JOURNAL OF THE

AMERICAN MATHEMATICAL SOCIETY

Volume 18, Number 2, Pages 253-289

S 0894-0347(05)00475-3

Article electronically published on January 3, 2005

\title{
THE AFFINE PLATEAU PROBLEM
}

\author{
NEIL S. TRUDINGER AND XU-JIA WANG
}

\section{INTRODUCTION}

In this paper we study the Plateau problem for affine maximal hypersurfaces, which is the affine invariant analogue of the classical Plateau problem for minimal surfaces. In particular we formulate the affine Plateau problem as a geometric variational problem for the affine area functional, and we prove the existence and regularity of maximizers. As a special case, we obtain corresponding existence and regularity results for the variational Dirichlet problem for the fourth order affine maximal surface equation, together with a uniqueness result for generalized solutions.

The affine Plateau problem may be formulated as follows. Let $\mathcal{M}_{0}$ be a bounded, connected hypersurface in Euclidean $(n+1)$-space, $\mathbf{R}^{n+1}$, with smooth boundary $\Gamma=\partial \mathcal{M}_{0}$. Assume that $\mathcal{M}_{0} \cup \Gamma$ is smooth and locally uniformly convex up to boundary. Let $S\left[\mathcal{M}_{0}\right]$ denote the set of locally uniformly convex hypersurfaces $\mathcal{M}$ with boundary $\Gamma$, which can be smoothly deformed from $\mathcal{M}_{0}$ in the family of locally uniformly convex hypersurfaces whose Gauss mapping images lie in that of $\mathcal{M}_{0}$. A hypersurface $\mathcal{M} \subset \mathbf{R}^{n+1}$ is called locally uniformly convex if it is a $C^{2}$ immersion of an $n$-manifold $\mathcal{N}$, whose principal curvatures are everywhere positive, and the Gauss mapping of $\mathcal{M}$ is the mapping $G: \mathcal{M} \rightarrow S^{n}$ which assigns to every point in $\mathcal{M}$ its unit normal vector. The affine metric (also called the Berwald-Blaschke metric) on a locally uniformly convex hypersurface $\mathcal{M}$ is defined by

$$
g=K^{-1 /(n+2)} I I,
$$

where $K$ is the Gauss curvature and $I I$ is the second fundamental form of $\mathcal{M}$. From the affine metric we have the affine area functional

$$
A(\mathcal{M})=\int_{\mathcal{M}} K^{1 /(n+2)}
$$

The affine Plateau problem is to determine a hypersurface $\mathcal{M} \in S\left[\mathcal{M}_{0}\right]$, maximizing the functional $A$ over $S\left[\mathcal{M}_{0}\right]$, that is,

$$
A(\mathcal{M})=\sup \left\{A\left(\mathcal{M}^{\prime}\right) \mid \mathcal{M}^{\prime} \in S\left[\mathcal{M}_{0}\right]\right\} .
$$

Received by the editors September 3, 2003.

2000 Mathematics Subject Classification. Primary 35J40; Secondary 53A15.

Key words and phrases. Affine Plateau problem, affine maximal hypersurface, affine area functional, affine maximal surface equation, variational problem, second boundary value problem, a priori estimates, strict convexity, interior regularity, Bernstein Theorem, Monge-Ampère measure, curvature measure, Gauss mapping, locally convex hypersurface, generalized Legendre transform.

This research was supported by the Australian Research Council.

(C)2005 American Mathematical Society Reverts to public domain 28 years from publication 
Recall that the classical Plateau problem is formulated as a variational minimization problem for the Euclidean area. The two dimensional affine Plateau problem was raised by Chern in his pioneering article [9]; see also Calabi [7]. For this we have the following solution (Theorems 3.1, 6.2, and 8.3).

Theorem A. There exists a smooth, locally uniformly convex hypersurface $\mathcal{M} \in$ $S\left[\mathcal{M}_{0}\right]$ solving the variational Plateau problem (1.3) in the two dimensional case, $n=2$, if and only if the image of the Gauss mapping of $\mathcal{M}_{0}$ does not cover any hemi-sphere.

In higher dimensions we shall prove that the affine Plateau problem is solvable in a generalized sense (Theorem 3.1), with solutions locally uniformly convex and smooth if they are locally strictly convex (Theorem 6.2).

A special case of the affine Plateau problem occurs when $\mathcal{M}_{0}$ is the graph of a smooth, locally uniformly convex function $\phi$ defined on a bounded smooth domain $\Omega \subset \mathbf{R}^{n}$. If $\mathcal{M}=\mathcal{M}_{u}$ is the graph of a locally convex function $u \in C^{2}(\Omega)$, the affine area of $\mathcal{M}$ is given by

$$
A(u):=A\left(\mathcal{M}_{u}\right)=\int_{\Omega}\left[\operatorname{det} D^{2} u\right]^{1 /(n+2)} .
$$

The functional $A$ in (1.4) is concave and upper semi-continuous with respect to local uniform convergence. If a smooth, locally uniformly convex function $u$ is stationary for $A$, then $u$ satisfies the affine maximal surface equation,

$$
L[u]:=U^{i j} w_{i j}=0,
$$

where

$$
w=\left[\operatorname{det} D^{2} u\right]^{-(n+1) /(n+2)},
$$

and $\left[U^{i j}\right]$ is the cofactor matrix of the Hessian matrix $D^{2} u$, which is divergence free for any fixed $i$ or $j$. The subscripts $i, j$ denote partial derivatives with respect to the variables $x_{i}, x_{j}$. Here $L$ is a nonlinear fourth order partial differential operator, which is elliptic with respect to locally uniformly convex solutions. The concavity of $A$ implies that the affine maximal surface equation (1.5) is both a necessary and sufficient condition for a smooth, locally uniformly convex function $u$ to locally maximize the affine area functional (1.4).

In the graph case the set $S\left[\mathcal{M}_{0}\right]=S[\phi](=S[\phi, \Omega])$ consists of locally uniformly convex functions $u \in C^{2}(\Omega) \cap C^{0}(\bar{\Omega})$ satisfying $u=\phi$ on $\partial \Omega$ and $D u(\Omega) \subset D \phi(\Omega)$. Accordingly we have the variational problem of finding a function $u \in S[\phi]$, maximizing the affine area functional $A$ on $S[\phi]$, that is,

$$
A(u)=\sup \{A(v) \mid v \in S[\phi]\},
$$

and as a special case of Theorem $\mathrm{A}$, this problem is solvable for $n=2$. More generally, we will extend the definition of affine area to nonsmooth locally convex functions (and locally convex hypersurfaces) in Section 2 and study the variational problem of maximizing the extended affine area $A$ on $\bar{S}[\phi]$, the closure of $S[\phi]$ under uniform convergence, that is, to find $u \in \bar{S}[\phi]$ such that

$$
A(u)=\sup \{A(v) \mid v \in \bar{S}[\phi]\} .
$$

We prove the existence of a unique maximizer $u$ for the extended functional (1.8), which is smooth if it is locally strictly convex (Theorems 2.1 and 6.2). 
Theorem B. There exists a unique locally convex function $u$ solving the variational boundary value problem (1.8), in all dimensions, which is smooth and locally uniformly convex in the interior of any set where it is locally strictly convex.

The variational problems (1.7) and (1.8) extend the first boundary value problem for the affine maximal surface equation (1.5),

$$
\begin{gathered}
u=\phi \text { on } \partial \Omega, \\
D u=D \phi \text { on } \partial \Omega,
\end{gathered}
$$

for if we have a classical, locally uniformly convex solution $u \in C^{4}(\Omega) \cap C^{1}(\bar{\Omega})$ of (1.5), (1.9), $u$ will also solve (1.7) uniquely. Note that if the domain $\Omega$ is convex, a locally convex function is convex, namely, its graph lies above its tangent planes everywhere.

The existence of at least nonsmooth maximizers in Theorems A and B follows from the upper semi-continuity of the affine area functional (1.4). For Theorem A we also need the fundamental lemma from [22] that a locally convex hypersurface with boundary on a hyperplane is convex, which facilitates the use of uniform local graph representations. The existence and uniqueness of the maximizers for the variational problem (1.8) will be proved in Section 2 (Theorem 2.1). For the uniqueness we need a preliminary result, Lemma 2.3, which guarantees that the Monge-Ampère measure of a maximizer is regular. The general Plateau problem is treated in Section 3, where we prove the existence of maximizers for the corresponding extended variational problem (1.3) in the class of locally convex hypersurfaces, under the necessary condition that the Gauss map of $\mathcal{M}_{0}$ does not cover any hemi-sphere (Theorem 3.1).

The rest of this paper, Sections 4 through 8 , deals with the issue of regularity. In Section 4 we reprove and extend to inhomogeneous equations

$$
L[u]=f
$$

a priori estimates for smooth, lcally uniformly convex solutions, already established in 21] (Theorems 4.1 and 4.2). These estimates are then employed to obtain corresponding regularity results through approximation by solutions to the second boundary value problem,

$$
\begin{array}{cc}
u=\phi & \text { on } \quad \partial \Omega, \\
w=\psi & \text { on } \quad \partial \Omega,
\end{array}
$$

which is the Dirichlet problem when (1.10) is considered as a second order system of two equations in $u$ and $w$. In Section 5 we prove the existence of loally smooth solutions to the boundary value problem (1.10), (1.11) (Theorem 5.1). Using a penalty method, we then prove in Section 6 that maximizers of the extended functional (1.8) can be approximated locally in $\Omega$ by smooth, uniformly convex solutions of the affine maximal surface equation (1.5) (Theorem 6.1). As consequences, we conclude a fundamental regularity result in all dimensions, that strictly convex affine maximal functions are smooth (Theorem 6.2), thereby completing the proof of Theorem B and extend our two dimensional Bernstein Theorem in [21] to general convex affine maximal functions (Theorem 6.3).

As with the Monge-Ampère equation, the strict convexity of solutions is a critical issue for the affine maximal surface equation. In [21] we proved the Bernstein theorem holds in any dimension under an asymptotic, strict convexity assumption, 
which is automatically satisfied if any affine maximal hypersurface with boundary on a hyperplane is strictly convex, which we proved for affine maximal surfaces in $\mathbf{R}^{3}$. In this paper we prove the maximizers in Theorems A and B are strictly convex for $n=2$ under general boundary conditions. The argument in this case becomes much more complicated than that in [21, since we have to eliminate the possibility of straight line segments in graphs having both endpoints at the boundary. In Section 7 we treat the extension of the Legendre transform to locally convex functions in arbitrary domains in preparation for our treatment of the strict convexity in Section 8. There we prove that in two dimensions the maximizers of the affine area functionals (1.3) and (1.8) are locally strictly convex, thus completing the proof of Theorem A (Theorems 8.2 and 8.3).

The inhomogeneous equation (1.10), which is crucial for our approximation arguments, is a prescribed affine mean curvature equation, since the quantity

$$
H_{A}[u]=-\frac{1}{n+1} L[u]
$$

is the affine mean curvature of the graph of $u$ [1], [16, [20, and an affine maximal graph is one with vanishing affine mean curvature. The corresponding variational problem to maximize the functional

$$
A_{f}(u)=A(u)-\int f u
$$

is actually treated in Section 2 for bounded $f$, with the inhomogeneous version of Theorem B proved in Theorems 2.1 and 6.2.

We conclude the paper with some remarks on the boundary behavior of maximizers and the regularity problem in higher dimensions.

\section{The AFFine AREA FUnCtionaL}

In this section, we prove the existence and uniqueness of maximizers for the variational problem (1.8). First we need to extend the affine area functional (1.4) to arbitrary locally convex functions and prove its upper semi-continuity. Note that since we will be including domains which are not convex, it is necessary to distinguish locally convex functions from convex functions. However, when there is no ambiguity, we will typically abbreviate locally convex to convex.

We begin with the definition of the Monge-Ampère measure. Let $\Omega$ be a bounded domain in $\mathbf{R}^{n}$ and $u$ a convex function in $\Omega$. The normal mapping of $u, N_{u}$, is a multi-valued mapping, defined as follows [17]. For any point $x \in \Omega, N_{u}(x)$ is the set of slopes of support hyperplanes of $u$ at $x$, that is,

$$
N_{u}(x)=\left\{p \in \mathbf{R}^{n} \mid u(y) \geq u(x)+p \cdot(y-x) \forall y \in \Omega\right\} ;
$$

and for any Borel set $E, N_{u}(E)=\bigcup_{x \in E} N_{u}(x)$. When $u$ is $C^{1}$, the normal mapping coincides with the gradient mapping $D u$.

From the normal mapping one introduces the Monge-Ampère measure $\mu[u]$, which is a Radon measure given by

$$
\mu[u](E)=\left|N_{u}(E)\right|,
$$

that is, the Lebesgue measure of $N_{u}(E)$. When $u$ is $C^{2}$, we have $\mu[u]=\left(\operatorname{det} D^{2} u\right) d x$.

It is a basic result of Aleksandrov that $\mu[u]$ is weakly continuous with respect to the convergence of convex functions. That is, if $\left\{u_{j}\right\}$ is a sequence of convex 
functions converging to $u_{0}$ in $L_{l o c}^{1}$, then $\mu\left[u_{j}\right]$ converges weakly to $\mu\left[u_{0}\right]$ as measures. It follows that for any closed set $E \subset \Omega$,

$$
\mu\left[u_{0}\right](E) \geq \lim _{j \rightarrow \infty} \mu\left[u_{j}\right](E) .
$$

The Monge-Ampère measure $\mu[u]$, as a Radon measure, can be decomposed as the sum of a regular part and a singular part. That is,

$$
\mu[u]=\mu_{r}[u]+\mu_{s}[u],
$$

where the regular part $\mu_{r}[u]$ is a measure given by a locally integrable function, $\mu_{r}[u]=\left(\mu_{r}[u]\right) d x$ (we use the same notation $\mu_{r}[u]$ to denote the measure and its density) and, the singular part $\mu_{s}[u]$ is a measure supported on a set with Lebesgue measure zero.

The regular part $\mu_{r}[u]$ is determined by the function $u$ explicitly. Indeed, since $u$ is convex, it is twice differentiable almost everywhere. In this paper we will use the notation

$$
\partial^{2} u=\left(\partial_{i j} u\right)
$$

to denote the Hessian matrix of a function if it is twice differentiable almost everywhere. Then $\operatorname{det} \partial^{2} u$ is a measurable function, and $\partial^{2} u=D^{2} u$ when $u$ is $C^{2}$ smooth.

Lemma 2.1. Let $u$ be a convex function. Then

$$
\mu_{r}[u]=\operatorname{det} \partial^{2} u \text {. }
$$

Proof. Let $u_{h}$ be the mollification of $u$. That is,

$$
u_{h}(x)=h^{-n} \int_{\Omega} u(y) \eta\left(\frac{x-y}{h}\right) d y
$$

for some nonnegative smooth function $\eta$ supported on the unit ball $B_{1}(0)$ and satisfying $\int_{\mathbf{R}^{n}} \eta=1$, where $x \in \Omega_{h}=\{x \in \Omega \mid \operatorname{dist}(x, \partial \Omega)>h\}$. Then at any point $x \in \Omega$ where $u$ is twice differentiable, we have $D^{2} u_{h}(x) \rightarrow \partial^{2} u(x)$ [25]. Hence for any measurable set $E \subset \subset \Omega$,

$$
\int_{E} \operatorname{det} \partial^{2} u \leq \lim _{h \rightarrow 0} \int_{E} \operatorname{det} D^{2} u_{h} .
$$

By the weak convergence (2.1), we have for any closed set $E \subset \Omega$ such that $\mu_{s}[u](E)=0$

$$
\int_{E} \operatorname{det} \partial^{2} u \leq \mu_{r}[u](E) .
$$

That is,

$$
\operatorname{det} \partial^{2} u \leq \mu_{r}[u] \quad \text { a.e. }
$$

We claim that the equality holds in (2.5). Indeed, for a.e. $x_{0} \in \Omega$ we have

$$
\begin{aligned}
\mu_{r}[u]\left(x_{0}\right) & =\lim _{\varepsilon \rightarrow 0} \frac{\mu_{r}[u]\left(B_{\varepsilon}\left(x_{0}\right)\right)}{\left|B_{\varepsilon}\left(x_{0}\right)\right|} \\
& \leq \lim _{\varepsilon \rightarrow 0} \frac{\mu[u]\left(B_{\varepsilon}\left(x_{0}\right)\right)}{\left|B_{\varepsilon}\left(x_{0}\right)\right|} .
\end{aligned}
$$

If $u$ is twice differentiable at $x_{0}$, then for any $x \in B_{\varepsilon}\left(x_{0}\right)$,

$$
\left|D u(x)-D u\left(x_{0}\right)-\partial^{2} u\left(x_{0}\right)\left(x-x_{0}\right)\right| \leq \delta\left|x-x_{0}\right|
$$


for some constant $\delta>0$, with $\delta \rightarrow 0$ as $\varepsilon \rightarrow 0$. Suppose for simplicity that $D u\left(x_{0}\right)=0$. Let $w=\frac{1}{2} x \cdot\left(\partial^{2} u\left(x_{0}\right)+\delta I\right) \cdot x$, where $I$ is the unit matrix. Then for $\left|x-x_{0}\right|$ sufficiently small, $N_{u}\left(B_{\varepsilon}\left(x_{0}\right)\right) \subset N_{w}\left(B_{\varepsilon}\left(x_{0}\right)\right)$. It follows that

$$
\begin{aligned}
\mu_{r}[u]\left(x_{0}\right) & \leq \lim _{\varepsilon \rightarrow 0} \frac{\left|N_{u}\left(B_{\varepsilon}\left(x_{0}\right)\right)\right|}{\left|B_{\varepsilon}\left(x_{0}\right)\right|} \\
& \leq \lim _{\varepsilon \rightarrow 0} \frac{\left|N_{w}\left(B_{\varepsilon}\left(x_{0}\right)\right)\right|}{\left|B_{\varepsilon}\left(x_{0}\right)\right|} \\
& =\operatorname{det}\left(\partial^{2} u\left(x_{0}\right)+\delta I\right) \\
& \leq \operatorname{det}\left(\partial^{2} u\left(x_{0}\right)\right)+C \delta,
\end{aligned}
$$

where $C$ depends on $\partial^{2} u\left(x_{0}\right)$ but is independent of $\varepsilon$. Sending $\varepsilon$ to zero, we obtain $\mu_{r}[u] \leq \operatorname{det} \partial^{2} u$ at $x_{0}$. Hence we have $\mu_{r}[u]=\operatorname{det} \partial^{2} u$.

Similarly for a given (nonsmooth) convex hypersurface $\mathcal{M}$ one can introduce the (Gauss) curvature measure on $\mathcal{M}$. Let $G: \mathcal{M} \rightarrow S^{n}$ denote the generalized Gauss mapping. That is, for any point $p \in \mathcal{M}, G(p)$ is the set of normals of the support hyperplanes of $\mathcal{M}$ at $p$. The curvature measure $\mu[\mathcal{M}]$ is defined, for any Borel set $E \subset \mathcal{M}$, by

$$
\mu[\mathcal{M}](E)=|G(E)| .
$$

The curvature measure can also be decomposed as the sum of a regular part and a singular part, namely,

$$
\mu[\mathcal{M}]=\mu_{r}[\mathcal{M}]+\mu_{s}[\mathcal{M}] .
$$

A similar proof as that of Lemma 2.1 shows that the regular part $\mu_{r}[\mathcal{M}]$ is given by the Gauss curvature of $\mathcal{M}$, which is well defined almost everywhere. In particular if $\mathcal{M}$ is the graph of a convex function $u$, then

$$
\mu_{r}[\mathcal{M}]=\frac{\operatorname{det} \partial^{2} u}{\left(1+|D u|^{2}\right)^{(n+2) / 2}} .
$$

By Lemma 2.1, we can extend the definition of the affine area functional (1.4) from smooth convex functions to general convex functions by

$$
A(u)=A(u, \Omega)=\int_{\Omega}\left[\operatorname{det} \partial^{2} u\right]^{1 /(n+2)} .
$$

By (2.7) we have

$$
A(\mathcal{M})=\int_{\mathcal{M}}\left(\mu_{r}[\mathcal{M}]\right)^{1 /(n+2)} .
$$

Formulae (2.8), (2.9) imply that the affine area is invariant under unimodular affine transformations in $\mathbf{R}^{n+1}$ and is independent of the choice of the coordinates. We remark that different but equivalent definitions for the affine surface area have been introduced [14]. Our definition (2.8) is more straightforward.

Defintion. A (locally) convex function $u$ in a domain $\Omega$ is called affine maximal if it is a maximizer of (2.8) under local convex perturbation. That is, for any (locally) convex function $v$ such that $u-v$ has compact support in $\Omega^{\prime} \subset \subset \Omega$, $A\left(v, \Omega^{\prime}\right) \leq A\left(u, \Omega^{\prime}\right)$. A locally convex hypersurface $\mathcal{M}$ is called affine maximal if locally it is the graph of an affine maximal function. 
Since the function $r \rightarrow(\operatorname{det} r)^{1 /(n+2)}$ is strictly concave on the cone of positive symmetric $n \times n$ matrices, so also is the functional $A$, that is,

$$
A(t u+(1-t) v) \geq t A(u)+(1-t) A(v)
$$

for all convex $u, v$ and $0 \leq t \leq 1$, with equality holding if and only if $\partial^{2} u=\partial^{2} v$. Moreover if $u$ is a convex function and $\eta$ is a continuous function twice differentiable a.e. such that $u+t \eta$ is convex for sufficiently small $t \geq 0$, we have

$$
\left.\frac{d}{d t} A(u+t \eta)\right|_{t=0}=\frac{1}{n+2} \int_{\Omega} w U^{i j} \partial_{i j} \eta
$$

where $w=\left(\operatorname{det} \partial^{2} u\right)^{-(n+1) /(n+2)}$, and $\left[U^{i j}\right]$ is the cofactor matrix of $\partial^{2} u$. Therefore a convex function is affine maximal if and only if for any such $\eta$ with compact support in $\Omega$,

$$
\int_{\Omega} w U^{i j} \partial_{i j} \eta \leq 0
$$

We will need the upper semi-continuity of the affine area functional, which was first proved in 15]; see also [21. Here we give a simple proof.

Lemma 2.2. Let $\left\{u_{m}\right\}$ be a sequence of convex functions in $\Omega$, converging locally uniformly to $u$. Then

$$
\limsup _{m \rightarrow \infty} A\left(u_{m}\right) \leq A(u) .
$$

Proof. By the Hölder inequality we have

$$
A(u, \Omega) \leq\left(\int \frac{\operatorname{det} \partial^{2} u}{\rho^{n+1}}\right)^{1 /(n+2)}\left(\int \rho\right)^{(n+1) /(n+2)}
$$

for any positive function $\rho$. It follows that

$$
\begin{aligned}
A(u, \Omega) & =\inf \left\{\left(\int \frac{\operatorname{det} \partial^{2} u}{\rho^{n+1}}\right)^{1 /(n+2)}, \rho \in C^{0}, \rho>0, \int \rho=1\right\} \\
& =\inf \left\{\left(\int \frac{d \mu_{r}[u]}{\rho^{n+1}}\right)^{1 /(n+2)}, \quad \rho i n C^{0}, \rho>0, \int \rho=1\right\} .
\end{aligned}
$$

Since the singular part is defined on a set of measure zero, we have

$$
A(u, \Omega)=\inf \left\{\left(\int \frac{d \mu[u]}{\rho^{n+1}}\right)^{1 /(n+2)}, \rho \in C^{0}, \rho>0, \int \rho=1\right\} .
$$

The upper semi-continuity then follows from the weak continuity of the MongeAmpère measure.

In the following we prove the existence and uniqueness of maximizers for (1.8). Let $\Omega$ be a bounded, Lipschitz domain in $\mathbf{R}^{n}$, and let $\phi$ be a convex function (not necessarily smooth) defined in a neighborhood of $\bar{\Omega}$. Denote by $\bar{S}[\phi, \Omega]$ the set of convex functions $v$ satisfying $v=\phi$ on $\partial \Omega$ and $N_{v}(\Omega) \subset N_{\phi}(\bar{\Omega})$. The latter relation means that if we extend $v$ to the neighborhood of $\bar{\Omega}$ such that $v=\phi$ outside $\Omega$, then $v$ is convex in the neighborhood of $\Omega$. Hence for any $\phi_{1} \in \bar{S}[\phi, \Omega]$, we have $\bar{S}\left[\phi_{1}, \Omega\right]=\bar{S}[\phi, \Omega]$ if we extend $\phi_{1}$ to a neighborhood of $\Omega$ by letting $\phi_{1}=\phi$. If $\phi$ is uniformly convex, then $\bar{S}[\phi, \Omega]$ is the closure of $S[\phi]$ under uniform convergence, where $S[\phi]$ is as introduced in Section 1. 
We will consider a more general maximization problem, that is,

$$
\sup \left\{A_{f}(v, \Omega) \mid v \in \bar{S}[\phi, \Omega]\right\}
$$

where

$$
A_{f}(v, \Omega)=A(v, \Omega)-\int_{\Omega} f v
$$

and $f \in L^{\infty}(\Omega)$ is a bounded, measurable function.

The existence of maximizers for (2.17) follows immediately from the upper semicontinuity of the affine area functional. To prove the uniqueness, we first show that a maximizer has nonsingular Monge-Ampère measure.

Lemma 2.3. Let $u$ be a maximizer of (2.17). Then the Monge-Ampère measure $\mu[u]$ has no singular part.

Proof. Suppose $\mu[u]$ has nonvanishing singular part $\mu_{s}[u]$. Since $\mu_{s}[u]$ is supported on a set of measure zero and $\mu_{r}[u]$ is an integral function, it follows that for any positive constant $K \geq 1$, there is a ball $B_{r} \subset \Omega$ such that

$$
\mu_{s}[u]\left(B_{r}\right) \geq K \mu_{r}[u]\left(B_{r}\right)+2 K^{2}\left|B_{r}\right|
$$

for otherwise by the covering lemma, $\mu_{s}$ must be an integrable function. Let $v$ be the solution to the Dirichlet problem

$$
\begin{aligned}
\mu[v] & =K \mu_{r}[u]+2 K^{2} \quad \text { in } \quad B_{r}, \\
v & =u \quad \text { on } \partial B_{r} .
\end{aligned}
$$

The existence and uniqueness of generalized solutions of the above problem are well known; see [17. Since $\mu[u]\left(B_{r}\right) \geq K \mu_{r}[u]\left(B_{r}\right)+2 K^{2}\left|B_{r}\right|$, the set $E=\{v>u\}$ is not empty. Let $\widetilde{u}=u$ in $\Omega-E$ and $\widetilde{u}=v$ in $E$. Then $\widetilde{u} \in \bar{S}[\phi, \Omega]$. We have

$$
\begin{aligned}
A_{f}(v, E)-A_{f}(u, E) & =\int_{E}\left(\operatorname{det} \partial^{2} v\right)^{1 /(n+2)}-\int_{E}\left(\operatorname{det} \partial^{2} u\right)^{1 /(n+2)}-\int_{E} f(v-u) \\
& \geq \int_{E}\left(K \operatorname{det} \partial^{2} u+2 K^{2}\right)^{1 /(n+2)}-\int_{E}\left(\operatorname{det} \partial^{2} u\right)^{1 /(n+2)}-C|E| .
\end{aligned}
$$

It is easy to see that the right-hand side is positive, by considering, respectively, the sets $\left\{\operatorname{det} \partial^{2} u>K\right\}$ and $\left\{\operatorname{det} \partial^{2} u<K\right\}$ and choosing $K$ sufficiently large. Hence we obtain

$$
A_{f}(\widetilde{u}, \Omega)=A_{f}(u, \Omega-E)+A_{f}(v, E)>A_{f}(u, \Omega) .
$$

It follows that $u$ is not affine maximal, a contradiction.

Theorem 2.1. Let $\Omega$ be a bounded, Lipschitz domain in $\mathbf{R}^{n}$. Suppose $\phi$ is a convex Lipschitz function defined in a neighborhood of $\bar{\Omega}$ and $f$ is a bounded measurable function. Then there is a unique maximizer $u$ for (2.17).

Proof. As remarked above, the existence follows from the upper semi-continuity of the affine area functional. To see the uniqueness, we observe that by the concavity of the affine area functional, if both $u$ and $v$ are maximizers, we have $\operatorname{det} \partial^{2} u=\operatorname{det} \partial^{2} v$ almost everywhere, and so $\mu[u]=\mu[v]$ since both of them have no singular part by Lemma 2.3. It follows that $u=v$ by the uniqueness of generalized solutions to the Monge-Ampère equation [17] (note that the strict convexity of $\Omega$ is not needed for this). 
Remark 2.1. Theorem 2.1 can be extended to the functional

$$
A_{f}(v, \Omega)=A(v, \Omega)-\int_{\Omega} f(x, v),
$$

where $f(x, t)$ is locally bounded in $\bar{\Omega} \times \mathbf{R}^{1}$, measurable in $x$ and convex in $t$. By the uniqueness in Theorem 2.1, one also sees that if $u_{k}, k=1,2, \cdots$, are maximizers of $\sup \left\{A_{f_{k}}(v, \Omega) \mid v \in \bar{S}\left[\phi_{k}, \Omega\right]\right\}$ and if $\phi_{k} \rightarrow \phi$ in $C^{1}(\bar{\Omega}), f_{k} \rightarrow f$ in $L^{\infty}(\Omega)$, then $u_{k} \rightarrow u$ and $u$ is the maximizer of (2.17).

\section{The general Plateau problem}

To study the maximization problem (1.3), we need to deal with nonsmooth, locally convex hypersurfaces. By definition, a locally convex hypersurface is the image of a locally convex immersion in $\mathbf{R}^{n+1}$ of a connected manifold $\mathcal{N}$, that is, $\mathcal{M}=T(\mathcal{N})$, on which there is a continuous vector field pointing everywhere to the convex side. This latter condition rules out hypersurfaces such as $x_{n+1}=$ $x_{1} \max \left(\left|x_{1}\right|-1,0\right)$. Recall that an immersion $T: \mathcal{N} \rightarrow \mathbf{R}^{n+1}$ is called locally convex if for any point $p \in \mathcal{N}$, there is a neighborhood $N_{\delta}(p) \subset \mathcal{N}$ such that $T\left(N_{\delta}(p)\right)$ is a convex graph in $\mathbf{R}^{n+1}$. We say a locally convex hypersurface $\mathcal{M}$ is convex if $\mathcal{M}$ lies in the boundary of its convex closure.

For any given point $x$ on a locally convex hypersurface $\mathcal{M}, T^{-1}(x)$ may contain more than one point in $\mathcal{N}$. To avoid confusion in the following, when referring to a point $x \in \mathcal{M}$, we need to understand a pair $(x, p)$, where $p=p_{x} \in \mathcal{N}$ such that $T(p)=x$. Also we say $\omega_{x} \subset \mathcal{M}$ is a neighborhood of $x$ if it is the image of a neighborhood of $p$ in $\mathcal{N}$. We say $\gamma$ is a curve on $\mathcal{M}$ if it is the image of a curve in $\mathcal{N}$ and so on. The $r$-neighborhood of $x, \omega_{r}(x)$, is the connected component of $\mathcal{M} \cap B_{r}(x)$ containing the point $x$.

As a prelude we proved in [22] a fundamental result for locally convex hypersurfaces, which plays a crucial role in our investigation of the affine Plateau problem.

Lemma 3.1. Let $\mathcal{M}$ be a compact, locally convex hypersurface in $\mathbf{R}^{n+1}, n>1$. Suppose the boundary $\partial \mathcal{M}$ lies in the hyperplane $\left\{x_{n+1}=0\right\}$. Then any connected component of $\mathcal{M} \cap\left\{x_{n+1}<0\right\}$ is convex.

Lemma 3.1 has two important applications to the affine Plateau problem, namely, to the existence of maximizers for (1.3) and to their strict convexity. Indeed, by Lemma 3.1 we conclude that the set $S\left[\mathcal{M}_{0}\right]$ is precompact, and so by the upper semi-continuity of the affine area functional, (1.3) admits a maximizer in $\bar{S}\left[\mathcal{M}_{0}\right]$, the closure of $S\left[\mathcal{M}_{0}\right]$ under local uniform convergence. For the strict convexity of the maximizer, Lemma 3.1 enables us to reduce consideration to the graph case (see the proof of Theorem 8.3).

To see that the set $S\left[\mathcal{M}_{0}\right]$ is precompact, we need a uniform cone property of locally convex hypersurfaces. Let $\mathcal{C}_{x, \xi, r, \alpha}$ denote the cone with vertex $x$, axis $\xi$, radius $r$, and aperture $\alpha$, that is,

$$
\mathcal{C}_{x, \xi, r, \alpha}=\left\{y \in \mathbf{R}^{n+1}|| y-x|<r,\langle y-x, \xi\rangle \geq \cos \alpha| y-x \mid\right\} .
$$

We say that $\mathcal{C}_{x, \xi, r, \alpha}$ is an inner contact cone of $\mathcal{M}$ at $x$ if this cone lies on the concave side of $\omega_{r}(x)$. We say $\mathcal{M}$ satisfies the uniform cone condition with radius $r$ and aperture $\alpha$ if $\mathcal{M}$ has an inner contact cone at all points with the same $r$ and $\alpha$. From Lemma 3.1, which we proved in [22], 
Lemma 3.2. Let $\mathcal{M} \subset B_{R}(0)$ be a locally convex hypersurface with boundary $\partial \mathcal{M}$. Suppose $\mathcal{M}$ can be extended to $\widetilde{\mathcal{M}}$ such that $\partial \mathcal{M}$ is embedded in $\widetilde{\mathcal{M}}$ and $\widetilde{\mathcal{M}}-\mathcal{M}$ is locally strictly convex. Then there exist $r, \alpha>0$ depending only on $n, R$, and the extended part $\widetilde{\mathcal{M}}-\mathcal{M}$ such that the $r$-neighborhood $\omega_{r}(x)$ is convex for any $x \in \mathcal{M}$, and $\mathcal{M}$ satisfies the uniform cone condition with radius $r$ and aperture $\alpha$.

The main point of Lemma 3.2 is that $r$ and $\alpha$ depend only on $n, R$ and the extended part $\widetilde{\mathcal{M}}-\mathcal{M}$. Therefore it holds with the same $r$ and $\alpha$ for a family of locally convex hypersurfaces, which includes all locally uniformly convex hypersurfaces, contained in $B_{R}(0)$, with boundary $\partial \mathcal{M}$ and Gauss mapping image coinciding with that of $\mathcal{M}$. For any sequence of locally convex hypersurfaces in this family, the uniform cone property implies that the sequence sub-converges and the limit hypersurface is locally a graph. This property was crucial for the Plateau problem for prescribed constant Gauss curvature [22] and also in the following existence proof of maximizers to the affine Plateau problem.

Let $\mathcal{M}_{0} \subset \mathbf{R}^{n+1}$ be a bounded hypersurface with smooth boundary which is smooth and locally uniformly convex up to its boundary $\Gamma$. As in 22 we extend $\mathcal{M}_{0}$ to a smooth, locally uniformly convex hypersurface $\widetilde{\mathcal{M}}_{0}$ such that $\Gamma$ lies in the interior of $\widetilde{\mathcal{M}}_{0}$. Denote $\mathcal{M}_{0}^{c}=\widetilde{\mathcal{M}}_{0}-\mathcal{M}_{0}$. As in Section 1 we denote by $S\left[\mathcal{M}_{0}\right]$ the set of locally uniformly convex hypersurfaces $\mathcal{M}$ with boundary $\Gamma$, which can be smoothly deformed from $\mathcal{M}_{0}$ in the family of locally uniformly convex hypersurfaces whose Gauss mapping images lie in that of $\mathcal{M}_{0}$. The latter assumption is equivalent to saying that $\mathcal{M} \cup \mathcal{M}_{0}^{c}$ is a locally convex hypersurface. Let $\bar{S}\left[\mathcal{M}_{0}\right]$ be the closure of $S\left[\mathcal{M}_{0}\right]$ under local uniform convergence. By Lemma 3.2, $\bar{S}\left[\mathcal{M}_{0}\right]$ is well defined. Moreover, any locally convex hypersurface in $\bar{S}\left[\mathcal{M}_{0}\right]$ satisfies the uniform cone condition with $r$ and $\alpha$ depending only on $n, R$ and $\mathcal{M}_{0}^{c}$. From Lemma 3.1, we have the following diameter estimate.

Lemma 3.3. If the image of the Gauss mapping of $\mathcal{M}_{0}$ does not cover any hemisphere in $S^{n}$, then there is $R>0$ such that $\mathcal{M} \subset B_{R}(0)$ for any $\mathcal{M} \in S\left[\mathcal{M}_{0}\right]$.

For the extended affine Plateau problem, we are concerned with the existence and regularity of maximizers to the problem

$$
\sup _{\mathcal{M} \in \bar{S}\left[\mathcal{M}_{0}\right]} A(\mathcal{M}) .
$$

Theorem 3.1. Let $\mathcal{M}_{0} \subset \mathbf{R}^{n+1}$ be a bounded hypersurface with smooth boundary which is smooth and locally uniformly convex up to its boundary $\Gamma$. Suppose the image of the Gauss mapping of $\mathcal{M}_{0}$ does not cover any hemi-sphere in $S^{n}$. Then there is a locally convex maximizer to (3.1).

Proof. The Gauss mapping image of $\mathcal{M}_{0}, \mathcal{N}$, is a locally uniformly convex hypersurface immersed in $S^{n}$ and can be decomposed into $m$ pieces, $\mathcal{N}=\bigcup_{i=1}^{m} F_{i}$, such that every piece is strictly contained in some hemi-sphere, namely, every $F_{i}$ is a graph with uniformly bounded gradient. For any $\mathcal{M} \in S\left[\mathcal{M}_{0}\right]$, let $\mathcal{M}^{(i)}=G^{-1}\left(F_{i}\right)$, where $G$ is the Gauss mapping of $\mathcal{M}$. Then $\mathcal{M}=\bigcup \mathcal{M}^{(i)}$ and $\mathcal{M}^{(i)}$ is a graph for any $i$.

It follows by Lemma 3.3 that any sequence in $S\left[\mathcal{M}_{0}\right]$ contains a convergent subsequence. Indeed, let $\left\{\mathcal{M}_{j}\right\}$ be a sequence in $S\left[\mathcal{M}_{0}\right]$. For any given $j$, as above we decompose $\mathcal{M}_{j}$ into the union of $\mathcal{M}_{j}^{(i)}, i=1, \cdots, m$. For each fixed $i$, we can 
choose a coordinate system such that $\mathcal{M}_{j}^{(i)}$ are graphs for all $j$. Hence by Lemma 3.3 and convexity, $\mathcal{M}_{j}^{(i)}$ contains a convergent subsequence. Moreover, by Lemma 3.3, $A\left(\mathcal{M}_{j}^{(i)}\right)$ is uniformly bounded for each $i$. Hence $A\left(\mathcal{M}_{j}\right)$ is uniformly bounded.

By the mollification of convex functions (see (2.3)) and the above decomposition, it is easy to see that

$$
\sup _{\mathcal{M} \in \bar{S}\left[\mathcal{M}_{0}\right]} A(\mathcal{M})=\sup _{\mathcal{M} \in S\left[\mathcal{M}_{0}\right]} A(\mathcal{M})<\infty .
$$

Hence the existence of maximizers to (3.1) follows from the upper semi-continuity of the affine area functional.

The necessity of the condition in Theorem $\mathrm{A}$, that is, if a hypersurface $\mathcal{M}$ is affine maximal, then the image of its Gauss mapping cannot contain any hemisphere, is readily shown. Indeed, if the Gauss mapping image contains the south hemi-sphere, we denote by $\mathcal{M}^{\prime}$ the preimage of the south hemi-sphere, given as a graph of a convex function $u$ over a domain $\Omega$. Then necessarily $\operatorname{det} D^{2} u \rightarrow \infty$ on $\partial \Omega$ and so $w \rightarrow 0$ on $\partial \Omega$. Applying the maximum principle to equation (1.5), regarding it as a linear, second order elliptic equation in $w$, we find that $w \equiv 0$ in $\Omega$. This is impossible. Also if the Gauss map image of $\mathcal{M}_{0}$ contains a hemi-sphere, then the functional in (3.1) is unbounded.

\section{A PRIORi estimates FOR CLASSiCAl SOlUtions}

If the maximizer in Theorem 2.1 is smooth and locally uniformly convex, it satisfies the nonlinear fourth order partial differential equation

$$
L[u]=f,
$$

where $L$ is the operator given in (1.5). In this section we establish a priori estimates for solutions of (4.1), as in 21.

Lemma 4.1. Let $u \in C^{4}(\Omega) \cap C^{0,1}(\bar{\Omega})$ be a locally uniformly convex solution of (4.1) with $u=0$ on $\partial \Omega$. Then, for any point $y \in \Omega$, we have the estimate

$$
\operatorname{det} D^{2} u(y) \leq C
$$

where $C$ depends on $n, \operatorname{dist}(y, \partial \Omega), \sup _{\Omega}|D u|, \sup _{\Omega} f$, and $\sup _{\Omega}|u|$.

Proof. Lemma 4.1 is proved in 21] (for the case $f \equiv 0$ ). We include the proof here for completeness.

Let

$$
z=\log \frac{w}{(-u)^{\beta}}-A|D u|^{2}
$$

where $\beta$ and $A$ are positive constants to be determined. Then $z$ attains its minimum at an interior point $x_{0} \in \Omega$. At $x_{0}$ we have

$$
\begin{aligned}
& 0=z_{i}=\frac{w_{i}}{w}-\beta \frac{u_{i}}{u}-2 A u_{k} u_{k i}, \\
& 0 \leq z_{i j}=\frac{w_{i j}}{w}-\frac{w_{i} w_{j}}{w^{2}}-\beta \frac{u_{i j}}{u}+\beta \frac{u_{i} u_{j}}{u^{2}}-2 A u_{k i} u_{k j}-2 A u_{k} u_{k i j}
\end{aligned}
$$

as a matrix. From $z_{i}=0$ we have

$$
\frac{w_{i} w_{j}}{w^{2}}=\beta^{2} \frac{u_{i} u_{j}}{u^{2}}+\frac{2 \beta A}{u}\left(u_{i} u_{k} u_{k j}+u_{j} u_{k} u_{k i}\right)+4 A^{2} u_{k} u_{l} u_{k i} u_{l j} .
$$


Using the identities

$$
\begin{aligned}
& u^{i j} u_{k i j}=-\frac{1}{1-\theta} \frac{w_{k}}{w}, \quad \theta=\frac{1}{n+2} \\
& u^{i j} w_{i j}=U^{i j} w_{i j} / d=f / d, \quad d=\operatorname{det} D^{2} u
\end{aligned}
$$

where $u^{i j}=U^{i j} / d$ is the inverse matrix of $D^{2} u$, we obtain

$$
\begin{aligned}
0 & \leq u^{i j} z_{i j} \\
& =\frac{f}{d^{\theta}}-\frac{\beta n}{u}-\frac{u^{i j} w_{i} w_{j}}{w^{2}}+\frac{\beta u^{i j} u_{i} u_{j}}{u^{2}}-2 A u^{i j} u_{k i} u_{k j}+\frac{2 A}{1-\theta} \frac{u_{k} w_{k}}{w} \\
& =\frac{f}{d^{\theta}}-\frac{\beta n}{u}-\beta(\beta-1) \frac{u^{i j} u_{i} u_{j}}{u^{2}}-2 A \Delta u+\frac{4 A^{2} \theta}{1-\theta} u_{i j} u_{i} u_{j}-2 \beta A \frac{1-2 \theta}{1-\theta} \frac{|D u|^{2}}{u} \\
& \leq \frac{f}{d^{\theta}}-A \Delta u-\frac{\beta n}{u}+2 \beta A \frac{|D u|^{2}}{|u|},
\end{aligned}
$$

with the choice

$$
A=\frac{1-\theta}{4 \theta \sup _{\Omega}|D u|^{2}}
$$

Therefore we have

$$
|u| \Delta u \leq C\left(1+|D u|^{2}\right) .
$$

Hence $z\left(x_{0}\right) \geq-C$ if we choose $\beta \geq n(n+1) /(n+2)$. It follows that $z(x) \geq z\left(x_{0}\right) \geq$ $-C$ and so (4.2) holds.

Remark 4.1. If $n=2$, the assumption $u=0$ on $\partial \Omega$ in Lemma 4.1 can be removed. Indeed, let

$$
z=\log \frac{w}{\eta^{\beta}}-A|D u|^{2},
$$

where $\eta(x)=\left(r^{2}-|x|^{2}\right)$ is a cut-off function and $r>0$ is such that $B_{r}(0) \subset \Omega$. Then similarly as above we have, at a maximum point of $z$,

$$
0 \leq u^{i j} z_{i j} \leq \frac{f}{d^{\theta}}-A \Delta u+\frac{\beta u^{i i}}{\eta}+2 \beta A \frac{u_{i} \eta_{i}}{\eta} .
$$

Note that

$$
u^{11}+u^{22}=\frac{u_{11}+u_{22}}{u_{11} u_{22}}=\frac{\Delta u}{d} .
$$

Hence we also obtain (4.2).

Remark 4.2. Lemma 4.1 holds if $f=f\left(x, u, D u, D^{2} u\right)$ and $f$ satisfies

$$
f(x, z, p, r) \leq C(1+\operatorname{tr} r)
$$

for any symmetric matrix $r$.

Lemma 4.2. Let $u \in C^{4}(\Omega) \cap C^{0,1}(\bar{\Omega})$ be a locally uniformly convex solution of (4.1). Suppose there exists an open set $\omega \subset \Omega$ such that $x \cdot D u<u$ in $\omega$ and $x \cdot D u=u$ on $\partial \omega$. Then for any $y \in \omega$,

$$
\operatorname{det} D^{2} u(y) \geq C,
$$

where $C>0$ depends on $n, \operatorname{dist}(y, \partial \omega), \sup _{\Omega}|D u|, \inf _{\Omega} f$ and $\sup _{\omega}|u-x \cdot D u|$. 
Proof. Let

$$
z=\log w+\beta \log (u-x \cdot D u)+A|x|^{2}
$$

for some positive constants $\beta$ and $A$ to be determined. Suppose $z$ attains its maximum at $x_{0} \in \omega$. Then at $x_{0}$,

$$
\begin{aligned}
& 0=z_{i}=\frac{w_{i}}{w}-\beta \frac{x_{k} u_{k i}}{\phi}+2 A x_{i}, \\
& 0 \geq z_{i i}=\frac{w_{i i}}{w}-\frac{w_{i}^{2}}{w^{2}}-\beta \frac{x_{k} u_{k i i}+u_{i i}}{\phi}-\beta \frac{x_{i}^{2} u_{i i}^{2}}{\phi^{2}}+2 A,
\end{aligned}
$$

where $\phi=u-x_{i} u_{i}$. By a rotation we may suppose $D^{2} u$ is diagonal at $x_{0}$. Then

$$
\begin{aligned}
0 & \geq u^{i i} z_{i i} \\
& =\frac{f}{d^{\theta}}-u^{i i}\left[\beta^{2} \frac{x_{i}^{2} u_{i i}^{2}}{\phi^{2}}-4 \beta A \frac{x_{i}^{2} u_{i i}}{\phi}+4 A^{2} x_{i}^{2}\right]-\frac{\beta x_{k}}{\phi} u^{i i} u_{k i i}-\frac{\beta n}{\phi}-\frac{\beta x_{i}^{2} u_{i i}}{\phi^{2}}+2 A u^{i i} \\
& =\frac{f}{d^{\theta}}-\beta(\beta+1) \frac{x_{i}^{2} u_{i i}}{\phi^{2}}+\frac{4 \beta A x_{i}^{2}}{\phi}+2 A\left(1-2 A x_{i}^{2}\right) u^{i i}-\frac{\beta n}{\phi}+\frac{\beta x_{i}}{\phi(1-\theta)} \frac{w_{i}}{w} \\
& =\frac{f}{d^{\theta}}-\frac{\beta n}{\phi}+\frac{4 \beta A x_{i}^{2}}{\phi}+A u^{i i}-\beta(\beta+1) \frac{x_{i}^{2} u_{i i}}{\phi^{2}}+\frac{\beta x_{i}}{\phi(1-\theta)}\left(\beta \frac{x_{i} u_{i i}}{\phi}-2 A x_{i}\right) \\
& \geq \frac{f}{d^{\theta}}-\frac{C}{\phi}+A u^{i i}+\beta\left(\frac{\beta}{1-\theta}-\beta-1\right) \frac{x_{i}^{2} u_{i i}}{\phi^{2}} \\
& \geq \frac{f}{d^{\theta}}-\frac{C}{\phi}+A u^{i i}
\end{aligned}
$$

if $\beta$ is large and $A$ is sufficiently small. It follows that $|\phi| u^{i i} \leq C$ and hence (4.4).

We note that Lemma 4.2 also follows from Lemma 4.1, using the Legendre transform, as in [21]; see equation (7.4). We can determine neighborhoods $\omega=\omega_{y}$ of points $y \in \Omega$ verifying the hypothesis of Lemma 4.2 in terms of a modulus of convexity of the strictly convex function $u$. For any $y \in \Omega, h>0$, we define the section $S_{h, u}^{0}(y)$ by

$$
S_{h, u}^{0}(y)=\{x \in \Omega \mid u(x)<u(y)+D u(y)(x-y)+h\} .
$$

The modulus of convexity of $u$ is a nonnegative function defined by

$$
\rho_{u}(r)=\inf _{y \in \Omega} \rho_{u, y}(r), \quad r>0,
$$

where

$$
\rho_{u, y}(r)=\sup \left\{h \geq 0 \mid S_{h, u}^{0}(y) \subset B_{r}(y)\right\}
$$

if there exists $h \geq 0$ such that $S_{h, u}^{0}(y) \subset B_{r}(y)$; otherwise we define $\rho_{u, y}(r)=0$. A general convex function $u$ is strictly convex if and only if $\rho_{u}(r)>0$ for all $r>0$.

If $u$ is a strictly convex solution of (4.1), we can characterize the open set $\omega_{y}$ $(y \in \Omega)$ in the following way. Let $\varepsilon>0$ be any given constant. Let $\mathcal{P}_{\varepsilon}$ denote the set of linear functions $g$ such that $g<u$ in $\Omega$ and $g(y)=u(y)-\varepsilon$. Let $\bar{g}(x)=\sup \left\{g(x) \mid g \in \mathcal{P}_{\varepsilon}\right\}$. Then $\bar{g} \leq u$ and the graph of $\bar{g}$ is a convex cone. Let $\omega$ denote the component of $\{\bar{g}<u\}$ containing $y$. Then if $\varepsilon<\rho_{u}\left(\frac{1}{2} r\right)$, where $r=\operatorname{dist}(y, \partial \Omega)$, we have $\bar{\omega} \subset \Omega$.

By Lemmas 4.1 and 4.2 we have the following Hölder and Sobolev space estimates. 
Theorem 4.1 ( $W^{4, p}$ estimate). Let $u \in C^{4}(\Omega)$ be a locally uniformly convex solution of (4.1). Then for any $\Omega^{\prime} \subset \subset \Omega, p \geq 1$, we have the estimate

$$
\|u\|_{W^{4, p}\left(\Omega^{\prime}\right)} \leq C,
$$

where $C$ depends on $n, p, \sup _{\Omega}|f|$, $\operatorname{dist}\left(\Omega^{\prime}, \partial \Omega\right)$, and the modulus of convexity of $u$.

Theorem 4.2 (Schauder estimate). Let $u \in C^{4}(\Omega)$ be a locally uniformly convex solution of (4.1) with $f \in C^{\alpha}(\bar{\Omega}), 0<\alpha<1$. Then $u \in C^{4, \alpha}(\Omega)$ and for any $\Omega^{\prime} \subset \subset \Omega$,

$$
\|u\|_{C^{4, \alpha}\left(\Omega^{\prime}\right)} \leq C,
$$

where $C$ depends on $n, \alpha,\|f\|_{C^{\alpha}(\Omega)}$, $\operatorname{dist}\left(\Omega^{\prime}, \partial \Omega\right)$, and the modulus of convexity of $u$.

To prove Theorems 4.1 and 4.2, we have, by Lemmas 4.1 and 4.2 and our control of the strict convexity of $u$,

$$
C_{1} \leq \operatorname{det} D^{2} u \leq C_{2}
$$

in any $\Omega^{\prime} \subset \subset \Omega$. Now we write (4.1) as a second order partial differential system

$$
\begin{aligned}
U^{i j} w_{i j} & =f \quad \text { in } \Omega, \\
\operatorname{det} D^{2} u & =w^{-(n+2) /(n+1)} \quad \text { in } \Omega .
\end{aligned}
$$

In [4] the authors proved, for the case $f \equiv 0$, a Hölder estimate, with respect to sections of $u$, for solutions $w$ of (4.9), assuming that the Monge-Ampère measure $\mu[u]$ satisfies a continuity condition with respect to the Lebesgue measure, which is guaranteed by (4.8). By examining their argument, one sees that under (4.8), their Hölder estimate extends to $f \in L^{\infty}$. Taking account of the modulus of convexity of $u$, we thus conclude the Hölder estimate for the function $w$. By the interior Schauder estimate of the Monge-Ampère equation [3], we then obtain interior Hölder estimates for the second derivatives of $u$. Hence (4.9) becomes a linear uniformly elliptic equation with Hölder continuous coefficients. It follows that we can estimate $w$ in $W_{l o c}^{2, p}(\Omega)$ for any $p<\infty$. The $W_{l o c}^{4, p}(\Omega)$ and $C^{4, \alpha}(\Omega)$ estimates for $u$ now follow from standard elliptic regularity theory.

Remark 4.3. Theorems 4.1 and 4.2 hold for any strictly convex solution of (4.1), by our regularity result, Theorem 6.2 .

To prove the regularity of maximizers in Theorems 2.1 and 3.1, it suffices to prove, in view of Theorems 4.1 and 4.2, that (a) the maximizers are strictly convex and (b) they can be approximated by smooth maximizers. We will prove (a) for dimension $n=2$ in Section 8 and (b) for all dimensions in Section 6 .

\section{The Second Boundary Value Problem}

In this section we prove the existence of solutions to the boundary value problem

$$
\begin{gathered}
L[u]=f(x, u) \quad \text { in } \Omega, \\
u=\phi \quad \text { on } \partial \Omega, \\
w=\psi \quad \text { on } \partial \Omega,
\end{gathered}
$$

where $L$ and $w$ are as in (1.5) (1.6), $\Omega$ is a smooth, uniformly convex domain in $\mathbf{R}^{n}, \phi, \psi$ are smooth functions on $\partial \Omega$ with

$$
C_{0}^{-1} \leq \psi \leq C_{0}
$$


for some positive constant $C_{0}$. We suppose $f \in L^{\infty}(\Omega \times \mathbf{R}), f$ is nondecreasing in $u$, and there is $t_{0} \leq 0$ such that

$$
f(x, t) \leq 0 \text { when } t \leq t_{0} .
$$

Theorem 5.1. The boundary value problem (5.1)-(5.3) admits a solution $u \in$ $W_{\text {loc }}^{4, p}(\Omega) \cap C^{0,1}(\bar{\Omega})(\forall p>1)$ with $\operatorname{det} D^{2} u \in C^{0}(\bar{\Omega})$. If $f \in C^{\alpha}(\bar{\Omega} \times \mathbf{R})$, where $\alpha \in(0,1)$, then the solution $u \in C^{4, \alpha}(\Omega) \cap C^{0,1}(\bar{\Omega})$.

We will use Theorem 5.1 in the next section to construct smooth approximations to the maximizers in Theorem 2.1.

To prove Theorem 5.1, we write (5.1) as a system (4.9), (4.10) and consider the approximating problem

$$
\begin{aligned}
U^{i j} w_{i j} & =f \quad \text { in } \Omega, \\
\operatorname{det} D^{2} u & =\eta w^{-(n+2) /(n+1)}+(1-\eta) \text { on } \Omega,
\end{aligned}
$$

where $u$ and $w$ satisfy the boundary condition (5.2) and (5.3), and $\eta=\eta_{k} \in C_{0}^{\infty}(\Omega)$ is a nonnegative cut-off function satisfying $\eta=1$ in $\Omega_{k}=\{x \in \Omega \mid \operatorname{dist}(x, \partial \Omega)<$ $1 / k\}$.

Lemma 5.1. Let $(u, w)$ be a $C^{2}$ smooth solution of (5.6)-(5.7). Then there exists a constant $C>0$ such that

$$
\begin{aligned}
C^{-1} \leq w & \leq C \quad \text { in } \Omega, \\
\left|w(x)-w\left(x_{0}\right)\right| & \leq C\left|x-x_{0}\right| \quad \forall x \in \Omega, x_{0} \in \partial \Omega,
\end{aligned}
$$

where $C$ depends only on $n, \operatorname{diam}(\Omega), \sup _{\Omega}|f|$, and $\sup _{\Omega}|u|$, and is independent of $k$.

Proof. Let $z=\log w-h(u)$, where $h$ is a convex, monotone increasing function satisfying $h(t)=t$ when $t \geq-t_{0}$ and $h(t) \geq-t_{0}-1$ when $t \leq-t_{0}$. If $z$ attains its minimum at a boundary point, by (5.4) we have $w \geq C$ in $\Omega$. If $z$ attains its minimum at an interior point $x_{0} \in \Omega$, we have

$$
\begin{aligned}
& 0=z_{i}=\frac{w_{i}}{w}-h^{\prime}(u) u_{i}, \\
& 0 \leq z_{i j}=\frac{w_{i j}}{w}-\frac{w_{i} w_{j}}{w^{2}}-h^{\prime}(u) u_{i j}-h^{\prime \prime}(u) u_{i} u_{j}
\end{aligned}
$$

as a matrix. Hence

$$
0 \leq u^{i j} z_{i j} \leq \frac{f}{d^{\theta}}-n h^{\prime}(u)
$$

where $d=\operatorname{det} D^{2} u, \theta=1 /(n+2)$. If $u\left(x_{0}\right) \leq t_{0}, f=0$ and we reach a contradiction. Hence $u\left(x_{0}\right) \geq t_{0}$ and $h^{\prime}(u)>0$. We obtain $d\left(x_{0}\right) \leq C$. Since $z(x) \geq z\left(x_{0}\right)$, we obtain

$$
w(x) \geq w\left(x_{0}\right) \exp \left(h(u(x))-h\left(u\left(x_{0}\right)\right)\right) .
$$

The first inequality in (5.8) follows.

Next let $z=\log w+A|x|^{2}$. If $z$ attains its maximum at a boundary point, by (5.4) we have $w \leq C$ and so (5.8) holds. If $z$ attains its maximum at an interior point $x_{0}$, we have, at $x_{0}$,

$$
\begin{aligned}
& 0=z_{i}=\frac{w_{i}}{w}+2 A x_{i}, \\
& 0 \geq z_{i i}=\frac{w_{i i}}{w}-\frac{w_{i}^{2}}{w^{2}}+2 A .
\end{aligned}
$$


Suppose $\left(D^{2} u\right)$ is diagonal at $x_{0}$. Then

$$
\begin{aligned}
0 & \geq u^{i j} z_{i j} \\
& =\frac{f}{d w}-4 A^{2} x_{i}^{2} u^{i i}+2 A u^{i i} \\
& \geq \frac{f}{d w}+A u^{i i}
\end{aligned}
$$

if $A$ is small. Hence

$$
d w \sum u^{i i} \leq C
$$

By (5.7) we obtain

$$
\left[\eta w^{-(n+2) /(n+1)}+(1-\eta)\right]^{(n-1) / n} w \leq d w \sum u^{i i} \leq C .
$$

We obtain $w \leq C$, and hence (5.8) is proved.

Let $v$ be a smooth, uniformly convex function in $\Omega$ such that $v=\psi$ on $\partial \Omega$ and $D^{2} v \geq K$. Then

$$
U^{i j} v_{i j} \geq K \sum U^{i i} \geq C K\left[\operatorname{det} D^{2} v\right]^{(n-1) / n} \geq C K .
$$

Hence if $K$ is large enough, $v$ is a lower barrier of $w$ by applying the comparison principle to (5.6). We thus obtain

$$
w(x)-w\left(x_{0}\right) \geq-C\left|x-x_{0}\right| \quad \forall x \in \Omega, x_{0} \in \partial \Omega .
$$

Similarly one can construct an upper barrier for $w$. Hence (5.9) holds.

By approximation, Lemma 5.1 holds for $w \in W^{2, p}(\Omega)$ with $p>n$. Indeed, let $\left\{f_{k}\right\}$ be a sequence of bounded functions which converges to $f$ in $L^{p}$, and let $w_{k}$ be the solution of (5.6) with $f=f_{k}$, where $U^{i j}$ is the cofactor matrix of $D^{2} u$, which is independent of $k$. Then $w_{k} \rightarrow w$ in $W^{2, p}$. As above we have the estimate $d w_{k} \sum u^{i i} \leq C$. Sending $k \rightarrow \infty$, we obtain (5.10) and so the second inequality in (5.8) follows. The first inequality in (5.8) can be proved in the same way as above.

Lemma 5.2. There is a solution $(u, w)$, where $u \in C^{2, \alpha}(\bar{\Omega})$ and $w \in W^{2, p}(\Omega)$ $(p>n)$, to the approximating problem (5.6), (6.7).

Proof. By (5.8), $u$ is uniformly bounded and is strictly convex in $\Omega$ [2. Applying the interior Hölder continuity result [4] to (5.6), we have $\operatorname{det} D^{2} u \in C^{\alpha}(\Omega)$ for some $\alpha \in(0,1)$, which in turn implies $w \in W^{2, p}(\Omega) \forall p>1$ and $u \in C^{2, \alpha}(\Omega)$. Near the boundary we also have $u \in C^{2, \alpha}$ by applying the regularity theory of the Monge-Ampère equation to (5.7) [5, 10, 13]. Therefore we have global estimates from which we can use degree theory to prove the existence of solutions.

For any positive $w \in C^{0,1}(\bar{\Omega})$, let $u=u_{w}$ be the solution of (5.7) with $u=\phi$ on $\partial \Omega$, and let $w_{t}, t \in[0,1]$, be the solution of

$$
\begin{aligned}
U^{i j} w_{i j} & =t f(x, u) \text { in } \Omega, \\
w_{t} & =t \psi+(1-t) \quad \text { on } \partial \Omega .
\end{aligned}
$$

Then the mapping $T_{t}: w \in C^{0,1}(\bar{\Omega}) \rightarrow w_{t} \in C^{0,1}(\bar{\Omega})$ is compact. By the above a priori estimates, the degree $\operatorname{deg}\left(T_{t}, B_{R}, 0\right)$ is well defined, where $B_{R}$ is the set of all positive function satisfying $\|w\|_{C^{0,1}(\bar{\Omega})} \leq R$. When $t=0$, from (5.12) we have obviously $w \equiv 1$. Namely, $T_{0}$ has a unique fixed point $w \equiv 1$. Hence the degree $\operatorname{deg}\left(T_{t}, B_{R}, 0\right)=1$ for all $t \in[0,1]$. This completes the proof. 
Denote by $u_{k}$ (corresponding to $\eta_{k}$ ) the solution obtained in Lemma 5.2, where the estimates for the upper and lower bounds of $\operatorname{det} D^{2} u_{k}$ are independent of $k$. Letting $u_{k} \rightarrow u$, we obtain (5.8) for $w=\left[\operatorname{det} D^{2} u\right]^{-(n+1) /(n+2)}$. Therefore we conclude $u \in W_{l o c}^{4, p}(\Omega) \cap C^{0,1}(\bar{\Omega})$ by Theorem 4.1. Note that by (5.8) we also have (5.9), which implies that $w \in C^{0}(\bar{\Omega})$ and $u$ satisfies the boundary conditions (5.2) and (5.3). If $f \in C^{\alpha}(\bar{\Omega} \times \mathbf{R})$, then $u \in C^{4, \alpha}(\Omega) \cap C^{0,1}(\bar{\Omega})$ by Theorem 4.2. Hence we obtain Theorem 5.1.

In a separate paper 23] we prove the uniqueness and global regularity of solutions to the boundary value problem (5.1)-(5.3).

\section{Approximation by Smooth solutions}

In this section we show that an affine maximal function can be approximated by smooth solutions of the affine maximal surface equation. Our approach also embraces the inhomogeneous case and utilizes the solvability of the second boundary value problem (Theorem 5.1).

We begin by considering a particular version of the second boundary value problem. Let $\phi \in C^{2}(\bar{B})$ be a uniformly convex function in a ball $B=B_{R}(0)$, vanishing on $\partial B$. Let $H \in C^{\infty}(-\infty, 1)$ be a nonnegative convex function such that

$$
H(t)= \begin{cases}4^{n}(1-t)^{-2 n} & \text { if } 1 / 2<t<1 \\ t^{4} & \text { if } t<-1\end{cases}
$$

Let $f \in L^{\infty}(\Omega)$ be the function in (2.18) and suppose $\Omega \subset \subset B$. Extend $f$ to $B$ such that

$$
f(x, u)=h(u-\phi(x)) \quad \text { in } B-\Omega,
$$

where $h(t)=H^{\prime}(t)$. Then $f$ is nondecreasing in $u$.

Lemma 6.1. There is a locally uniformly convex solution u to the boundary value problem

$$
\begin{aligned}
L[u] & =f(x, u) \text { in } B, \\
u & =\phi \quad \text { on } \partial B \\
w & =1 \quad \text { on } \partial B
\end{aligned}
$$

with $u \in W_{\text {loc }}^{4, p}(B) \cap C^{0,1}(\bar{B})$, for all $p<\infty, w \in C^{0}(\bar{\Omega})$, where $L$ is the operator in (1.5).

Proof. We will prove that if $u$ is a locally uniformly convex solution of (6.3), then

$$
\inf _{B} u \geq-K_{0}
$$

for some $K_{0}>0$ depending only on $\phi$ and $R$, the radius of the ball $B$; and

$$
|f(x, u)| \leq C .
$$

Once (6.4) and (6.5) are established, Lemma 6.1 follows from Theorem 5.1. 
First we prove (6.4). Let $\delta>0$ be a small constant. Since $\phi$ vanishes on $\partial B$, $\Omega_{\delta}=\{u<-\delta\}$ is strictly contained in $B$. We compute

$$
\begin{aligned}
\int_{\Omega_{\delta}} U^{i j} w_{i j}(u+\delta) & =-\int_{\Omega_{\delta}}(u+\delta)_{i} w_{j} U^{i j} \\
& =-\int_{\partial \Omega_{\delta}}(u+\delta)_{i} \gamma_{j} w U^{i j}+\int_{\Omega_{\delta}} w U^{i j}(u+\delta)_{i j} \\
& <n \int_{B} w \operatorname{det} D^{2} u=n \int_{B}\left[\operatorname{det} D^{2} u\right]^{\theta},
\end{aligned}
$$

where $\theta=1 /(n+2), \gamma$ is the unit outward normal, and we have used the divergence free property of $\left[U^{i j}\right]$ for any fixed $i$ or $j$. Sending $\delta$ to 0 , we obtain

$$
\int_{B} f(x, u) u \leq n \int_{B}\left[\operatorname{det} D^{2} u\right]^{\theta}=n \int_{\mathcal{M}} K^{\theta},
$$

where $\mathcal{M}$ is the graph of $u$ and $K$ is the Gauss curvature of $\mathcal{M}$. It follows that

$$
\int_{B} f(x, u) u \leq n|\mathcal{M}|^{1-\theta}\left[\int_{\mathcal{M}} K\right]^{\theta} \leq C|\inf u|^{1-\theta} .
$$

Recalling that $f$ is bounded in $\Omega$, we obtain

$$
\int_{B-\Omega} f(x, u) u \leq C|\inf u| .
$$

Since $u$ is convex and $u=0$ on $\partial B$, there exists $C>0$ such that

$$
\left|\inf _{B-\Omega} u\right|^{4} \geq C \int_{B}|u|^{4} .
$$

It follows by our construction of $H$ (see (6.1)) that

$$
\int_{B-\Omega} f(x, u) u \geq C_{1}|\inf u|^{4}-C_{2}
$$

for some positive constants $C_{1}, C_{2}>0$. Hence (6.4) holds.

Next we prove (6.5). Since $f(x, t)$ is increasing in $t, f(x, u)$ is bounded from below by (6.4). If suffices to prove that $f(x, u)$ is bounded from above.

We first prove $\operatorname{det} D^{2} u$ is bounded near $\partial B$. Indeed, by convexity and our choice of $H, f(x, u)$ is bounded from above near $\partial B$. For any boundary point $x_{0} \in \partial B$, we suppose by a rotation of axes that $x_{0}=(R, 0, \cdots, 0)$. Let $\ell(x)=a x_{1}+b$ be a linear function such that $\ell\left(x_{0}\right)<u\left(x_{0}\right)=0$ and $\ell>u$ on $x_{1}=R-\delta_{0}$, where $\delta_{0}>0$ is a constant such that $f$ is upper bounded in $B \cap\left\{x_{1}>R-\delta_{0}\right\}$. Let $z=\log \frac{w}{u-\ell}$. If $z$ attains a minimum at a boundary point $\partial B$, by the boundary condition $w=1$ in (6.3) we see that $z$ is bounded from below and so $\operatorname{det} D^{2} u$ is bounded from above near $\partial \Omega$. If $z$ attains a minimum at some interior point $y_{0} \in\{u>\ell\}$, we compute, at $y_{0}$,

$$
\begin{aligned}
0=z_{i} & =\frac{w_{i}}{w}-\frac{(u-\ell)_{i}}{u-\ell}, \\
z_{i j} & =\frac{w_{i j}}{w}-\frac{(u-\ell)_{i j}}{u-\ell}
\end{aligned}
$$

with the matrix $\left[z_{i j}\right] \geq 0$. It follows that

$$
0 \leq u^{i j} z_{i j}=\frac{f}{\left[\operatorname{det} D^{2} u\right]^{\theta}}-\frac{n}{u-\ell} .
$$


Hence $\operatorname{det} D^{2} u \leq C$ at $y_{0}$ and so $z \geq C$, which in turn implies that $\operatorname{det} D^{2} u$ is bounded near $\partial B$.

By (6.4) we then conclude that $u$ is Lipschitz at $\partial B$, and hence $D u$ is uniformly bounded in $B$ by convexity. Returning to (6.6), we have

$$
\begin{aligned}
\int_{\Omega_{\delta}} U^{i j} w_{i j}(u+\delta) & \geq-\int_{\partial \Omega_{\delta}} u_{i} \gamma_{j} w U^{i j} \\
& =-\int_{\partial \Omega_{\delta}} u_{\gamma} w U^{\gamma \gamma} \\
& =-\int_{\partial \Omega_{\delta}}\left(u_{\gamma}\right)^{n} w K_{s} \\
& \geq-n \omega_{n}\left(\sup _{B}|D u|\right)^{n} \inf _{\partial \Omega_{\delta}} w
\end{aligned}
$$

where $K_{s}$ denotes the Gauss curvature of $\partial \Omega_{\delta}$. Letting $\delta \rightarrow 0$, we obtain from (6.3),

$$
\int_{B}(-f(x, u)) u \leq C
$$

since $w=1$ on $\partial B$.

If $u(x)-\phi(x)$ is sufficiently close to one at some point $x \in B-\Omega$, then $u(x)-\phi(x)$ is sufficiently close to one at nearby points in $B-\Omega$, by the convexity of $u$ and $\phi$, and the integral on the left-hand side of (6.7) can be arbitrarily large, in contradiction with the estimate (6.7). Hence (6.5) holds. Lemma 6.1 now follows from Theorem 5.1 .

We remark that the function $f(x, u)$ is not defined when $u \geq \phi+1$. This is not a problem for the use of the degree argument in the proof of Lemma 5.2. One can also choose a sequence $f_{j}(x, u)$ which is defined for all $u \in \mathbf{R}$ and converges to $f$.

Next we use Lemma 6.1 and the penalty method to prove the maximizer in Theorem 2.1 can be approximated locally by smooth local maximizers.

Theorem 6.1. Let $\Omega$ and $\phi$ be as in Theorem 2.1. Then for any convex $\Omega^{\prime} \subset \subset \Omega$, there exists a sequence of smooth solutions of equation $(4.1)\left(\in W_{l o c}^{4, p}(\Omega) \forall p<\infty\right)$ converging uniformly in $\Omega^{\prime}$ to the maximizer $u$.

Proof. Without loss of generality let us assume that $\Omega$ is convex, $u$ is a maximizer in $\Omega^{\delta}=\left\{x \in \mathbf{R}^{n} \mid \operatorname{dist}(x, \Omega)<\delta\right\}$ for some $\delta>0$ small, $\phi=u$ in $\Omega^{\delta}-\Omega$. We will prove that $u$ can be approximated by smooth solutions of (4.1) in $\Omega$.

Let $B_{R}=B_{R}(0)$ be a ball in $\mathbf{R}^{n}$ containing $\overline{\Omega^{\delta}}$. Let

$$
\widetilde{\phi}(x)=\sup _{v \in \mathcal{P}} v(x), \quad x \in B_{R},
$$

where $\mathcal{P}$ is the set of linear functions $v$ such that $v \leq \phi$ in $\Omega^{\delta}$ and $v \leq K_{0}$ in $B_{R}$ for some given constant $K_{0}$. Since $\phi$ is Lipschitz and $\Omega$ is convex, we can choose $K_{0}$ sufficiently large such that $\widetilde{\phi}=\phi$ in $\Omega^{\delta}$. By definition, $\widetilde{\phi}$ cannot be strictly convex at any point in $B_{R}-\overline{\Omega^{\delta}}$. By Aleksandrov's theorem, the set $N_{\widetilde{\phi}}\left(B_{R}-\overline{\Omega^{\delta}}\right)$ has measure zero, that is, $\mu[\tilde{\phi}]=0$ in $B_{R}-\overline{\Omega^{\delta}}$. Therefore we may suppose directly that $\phi$ is defined in $B_{R}$ such that $\mu[\phi]=0$ in $B_{R}-\overline{\Omega^{\delta}}$ and $\phi$ equals the constant $K_{0}$ on $\partial B_{R}$.

Let $\left\{\phi_{k}\right\}$ be a sequence of convex functions such that $\phi_{k}=\phi$ in $\overline{\Omega^{\delta}}, \phi_{k}=\phi$ on $\partial B_{R}, \phi_{k}$ is uniformly convex in $\bar{B}_{R}-\overline{\Omega^{\delta}}$, and $\phi_{k} \rightarrow \phi$ uniformly in $\bar{B}_{R}$. Let 
$H_{j}(t)=H\left(2^{j} t\right)$ be a sequence of smooth, convex functions, defined in $\left(-\infty, 2^{-j}\right)$. Let $f_{k, j}(x, u)=f(x)$ when $x \in \Omega$ and $f_{k, j}(x, u)=h_{j}\left(u-\phi_{k}\right)$ when $x \in B_{R}-\Omega$, where $h_{j}=H_{j}^{\prime}$ and $f$ is the function in (2.17).

By Lemma 6.1 , there is a convex solution $u_{k, j}$ of $(6.3)$ with $f=f_{k, j}$, which is an extremal of the concave functional

$$
J_{k, j}(u)=J_{k, j}\left(u, B_{R}\right)=A\left(u, B_{R}\right)-\int_{\Omega} f u-\int_{B_{R}-\Omega} H_{j}\left(u-\phi_{k}\right),
$$

where $A\left(u, B_{R}\right)$ is the affine area functional on the domain $B_{R}$.

Similarly to (6.4) we have $u_{k, j} \geq-K_{0}$ for some $K_{0}$ independent of $k, j$. We have indeed a stronger estimate, for any given $k$,

$$
\inf _{B_{R}-\Omega}\left(u_{k, j}-\phi_{k}\right) \rightarrow 0 \quad \text { as } j \rightarrow \infty
$$

To prove (6.8), we suppose $\inf _{B_{R}-\Omega}\left(u_{k, j}-\phi_{k}\right)$ is attained at $x_{j}$. Let $\ell$ be the tangent plane of $\phi_{k}$ at $x_{j}$. Let $\omega=\left\{u_{k, j}<\ell\right\}$. We compute

$$
\begin{aligned}
\int_{\omega} U^{i j} w_{i j}(u-\ell) & =-\int_{\omega}(u-\ell)_{i} w_{j} U^{i j} \quad\left(u=u_{k, j}\right) \\
& =-\int_{\partial \omega}(u-\ell)_{i} \gamma_{j} w U^{i j}+\int_{\omega}(u-\ell)_{i j} w U^{i j} .
\end{aligned}
$$

The first integral on the right-hand side is negative. Hence we obtain

$$
\begin{aligned}
\int_{\omega} f_{k, j}(x, u)(u-\ell) & =\int_{\omega} U^{i j} w_{i j}(u-\ell) \\
& \leq \int_{\omega} w \operatorname{det} D^{2} u \\
& =\int_{\omega}\left(\operatorname{det} D^{2} u\right)^{\theta} \\
& =\int_{\mathcal{M}} K^{\theta} \leq C,
\end{aligned}
$$

where $\mathcal{M}$ is the graph of $u$ and $K$ is the Gauss curvature. If (6.8) is not true, the integral on the left-hand side converges to infinity (as $j \rightarrow \infty$ ) by our definition of $f_{k, j}$, which is a contradiction. Hence (6.8) holds.

Observe that by our definition of $H_{j}$,

$$
u_{k, j} \leq \phi_{k}+2^{-j}
$$

which, together with (6.8), implies that

$$
u_{k, j} \rightarrow \phi_{k}
$$

in $B_{R}-\Omega$ as $j \rightarrow \infty$.

Since $\phi_{k} \rightarrow \phi$, we have $u_{k, j} \rightarrow \phi(k, j \rightarrow \infty)$ locally uniformly in $B_{R}-\Omega$ as long as $j$ is large enough. By convexity, $u_{k, j}$ sub-converges to a convex function $\bar{u}$ in $B_{R}$. By our definition of $\bar{S}[\phi, \Omega]$ in Section 2 , the function $\bar{u}$, when restricted to $\Omega$, belongs to the set $\bar{S}[\phi, \Omega]$. We want to prove that $\bar{u}$ is the maximizer of $(2.17)$, whence $\bar{u}=u$ by the uniqueness assertion in Theorem 2.1.

Choose $R^{\prime}<R$ and $r>0$ such that $\Omega \subset B_{R^{\prime}-3 r}(0)$. Denote

$$
D_{\sigma}=\left\{x \in B_{R}(0)\left|R^{\prime}-r-\sigma<\right| x \mid<R^{\prime}+r+\sigma\right\}
$$


where $0 \leq \sigma \leq r$. Let

$$
\bar{u}_{k, j}=\sup \left\{v \mid v \in \Phi_{k, j}\right\},
$$

where $\Phi_{k, j}$ is the set of convex functions in $B_{R}(0)$ which satisfy $v \leq u_{k, j}$ in $B_{R}-D_{\sigma}$, $v \leq \max \left(\phi_{k}, u_{k, j}\right)$ in $D_{\sigma}$, and $v \leq \phi_{k}$ in $D_{0}=D_{\sigma \mid \sigma=0}$. Then for any fixed $r>\sigma>0$, by the uniform convexity of $\phi_{k}$ and (6.10), we have

$$
\begin{aligned}
& \bar{u}_{k, j}=u_{k, j} \quad \text { in } B_{R}-D_{\sigma}, \\
& \bar{u}_{k, j}=\phi_{k} \quad \text { in }\left\{x \in D_{0} \mid \operatorname{dist}\left(x, \partial D_{0}\right)>\sigma / 2\right\}, \\
& \left|\bar{u}_{k, j}-\phi_{k}\right| \leq\left|u_{k, j}-\phi_{k}\right| \quad \text { in } D_{\sigma}
\end{aligned}
$$

provided $j$ is sufficiently large. By $(6.11), \bar{u}_{k, j} \in \bar{S}\left[u_{k, j}, B_{R}\right]$. Since $u_{k, j}$ is the maximizer of $J_{k, j}$ in $\bar{S}\left[u_{k, j}, B_{R}\right]$, we have

$$
J_{k, j}\left(\bar{u}_{k, j}\right) \leq J_{k, j}\left(u_{k, j}\right) .
$$

Observe that

$$
\begin{aligned}
\int_{D_{\sigma}}\left[\operatorname{det} D^{2} u\right]^{1 /(n+2)} & \leq\left|D_{\sigma}\right|^{(n+1) /(n+2)}\left|\int_{B_{R}} \operatorname{det} D^{2} u\right|^{1 /(n+2)} \\
& \leq C\left|D_{\sigma}\right|^{(n+1) /(n+2)}
\end{aligned}
$$

where $u=u_{k, j}$. By (6.12) we have

$$
J_{k, j}\left(\bar{u}_{k, j}\right) \geq J_{k, j}\left(u_{k, j}\right)-\varepsilon
$$

with $\varepsilon \rightarrow 0$ as $r, \sigma \rightarrow 0$.

Let

$$
v_{k, j}= \begin{cases}u & \text { in } \Omega^{\delta}, \\ \phi_{k} & \text { in } B_{R^{\prime}}-\Omega^{\delta}, \\ \bar{u}_{k, j} & \text { in } B_{R}-B_{R^{\prime}}\end{cases}
$$

be an extension of $u$ in $B_{R}$. Then $v_{k, j}=u=\phi$ in $\Omega^{\delta}-\Omega$, and $v_{k, j} \in \bar{S}\left[u_{k, j}, B_{R}\right]$. Hence we have, by (6.13), (6.14),

$$
J_{k, j}\left(v_{k, j}\right) \leq J_{k, j}\left(u_{k, j}\right) \leq J_{k, j}\left(\bar{u}_{k, j}\right)+\varepsilon .
$$

Since $v_{k, j}=\bar{u}_{k, j}$ in $B_{R}-B_{R^{\prime}}$, we obtain

$$
J_{k, j}\left(v_{k, j}, B_{R^{\prime}}\right) \leq J_{k, j}\left(\bar{u}_{k, j}, B_{R^{\prime}}\right)+\varepsilon .
$$

Since $H_{k, j} \geq 0$, we have

$$
\begin{aligned}
J_{k, j}\left(\bar{u}_{k, j}, B_{R^{\prime}}\right) & \leq A\left(\bar{u}_{k, j}, B_{R^{\prime}}\right)-\int_{\Omega} f \bar{u}_{k, j} \\
& =A_{f}\left(\bar{u}_{k, j}, \Omega\right)+A\left(\bar{u}_{k, j}, B_{R^{\prime}}-\Omega\right),
\end{aligned}
$$

where

$$
A_{f}(u, \Omega)=A(u, \Omega)-\int_{\Omega} f u .
$$


We compute

$$
\begin{aligned}
A\left(\bar{u}_{k, j}, B_{R^{\prime}}-\Omega\right)= & A\left(\bar{u}_{k, j}, \Omega^{\delta}-\Omega\right)+A\left(\bar{u}_{k, j}, B_{R^{\prime}}-\Omega^{\delta}\right) \\
\leq & \left|\Omega^{\delta}-\Omega\right|^{(n+1) /(n+2)}\left[\int_{\Omega^{\delta}-\Omega} \operatorname{det} D^{2} \bar{u}_{k, j}\right]^{1 /(n+2)} \\
& +C\left[\int_{B_{R^{\prime}}-\Omega^{\delta}} \operatorname{det} D^{2} \bar{u}_{k, j}\right]^{1 /(n+2)} .
\end{aligned}
$$

We choose $\delta>0$ sufficiently small such that the first term on the right-hand side is small. Observe that $\bar{u}_{k, j} \rightarrow \phi$ and the Monge-Ampère measure $\mu[\phi]=0$ in $B_{R}-\Omega^{\delta}$. We therefore obtain

$$
A\left(\bar{u}_{k, j}, B_{R^{\prime}}-\Omega\right) \leq 2 \varepsilon
$$

if $k$ is large enough. Therefore by (6.15), (6.16), and the upper semi-continuity of the affine area functional, we obtain

$$
\begin{aligned}
A_{f}(u, \Omega) & \leq J_{k, j}\left(v_{k, j}, B_{R^{\prime}}\right) \\
& \leq J_{k, j}\left(\bar{u}_{k, j}, B_{R^{\prime}}\right)+\varepsilon \\
& \leq A_{f}\left(\bar{u}_{k, j}, \Omega\right)+2 \varepsilon \\
& \leq A_{f}(\bar{u}, \Omega)+3 \varepsilon
\end{aligned}
$$

if $k, j$ are sufficiently large. Hence $\bar{u}$ is the maximizer, and thus $\bar{u}=u$.

The penalty method is used above to create a sequence of smooth solutions of (4.1) satisfying (6.10). The function $H$, chosen in (6.1), facilitates the estimate (6.9). This function can be replaced by convex functions defined in $(-\infty, \infty)$ which grow much faster for $t>0$ than for $t<0$.

From Theorem 6.1 we obtain the following interior regularity in all dimensions, which includes the case of affine maximal functions and thus completes the proof of Theorem B.

Theorem 6.2. Let $u$ be a strictly convex maximizer of (2.17), with $f \in C^{\infty}(\Omega)$. Then $u \in C^{\infty}(\Omega)$ and satisfies equation (4.1) in $\Omega$.

In the case of an affine maximal graph $\mathcal{M}=\mathcal{M}_{u}$ (that is $f \equiv 0$ ), we obtain from Theorem 6.1 and the uniqueness part of Theorem 2.1 that there exists a sequence of smooth functions $\left\{u_{m}\right\} \subset C^{\infty}(\Omega)$ with affine maximal graphs, converging locally uniformly to $u$. As a byproduct, we may extend our affine Bernstein result in [21] to nonsmooth affine maximal surfaces.

Theorem 6.3. Let $u$ be an affine maximal convex function defined in the whole space $\mathbf{R}^{2}$. Suppose $u$ has at least one strictly convex point. Then $u$ is a quadratic function.

The assumption that $u$ has at least one strictly convex point implies that the level set $S_{h, u}^{0}(p)$ is a bounded convex domain for some point $p \in \mathbf{R}^{2}$. Note that if $u$ has no strictly convex point, then $u\left(x_{1}, x_{2}\right)=\phi\left(x_{1}\right)$ for some convex function $\phi$ in an appropriate coordinate system.

In the following two sections we will show that the hypothesis of strict convexity can be dispensed with in two dimensions. 


\section{THE GENERALIZED LEGENDRE TRANSFORM}

In this section we introduce the Legendre transform for (nonsmooth, locally) convex functions in general domains, which will be used in the next section to prove the strict convexity of maximizers in Theorems 2.1 and 3.1.

Let $\Omega$ be a convex domain and let $u \in C^{2}(\bar{\Omega})$ be a uniformly convex function. The Legendre transform of $u$ is the function $u^{*}$ defined in $\Omega^{*}=D u(\Omega)$, given by

$$
\begin{aligned}
u^{*}(y) & =\sup \{\widetilde{x} \cdot y-u(\widetilde{x}) \mid \widetilde{x} \in \Omega\} \\
& =x \cdot y-u(x),
\end{aligned}
$$

where $x \in \Omega$ is uniquely determined by $y=D u(x)$. The Legendre transform $u^{*}$ is a uniformly convex, $C^{2}$ smooth function in $\Omega^{*}$. Furthermore the Legendre transform of $u^{*}$ is $u$ itself.

From the relation $y=D u(x)$ we have $x=D u^{*}(y)$ and

$$
\left\{D^{2} u(x)\right\}=\left\{D^{2} u^{*}(y)\right\}^{-1} \text {. }
$$

Therefore if $u \in C^{2}(\bar{\Omega})$ is affine maximal, namely, if it is a maximizer of the affine area functional $A$ in the set $\bar{S}[u, \Omega], u^{*}$ is a maximizer of the associated functional

$$
A^{*}(v)=\int_{\Omega^{*}}\left[\operatorname{det} \partial^{2} v\right]^{(n+1) /(n+2)}
$$

in the set $\bar{S}\left[u^{*}, \Omega^{*}\right]$. If $u$ is a smooth, uniformly convex solution of the nonhomogeneous equation (4.1), then by direct computation, $u^{*}$ satisfies the equation

$$
U^{i j} w_{i j}^{*}=-\frac{1}{n+1} f\left(D u^{*}\right) \operatorname{det} D^{2} u^{*},
$$

where $U^{i j}$ is the cofactor matrix of $D^{2} u^{*}$ and $w^{*}=\left[\operatorname{det} D^{2} u^{*}\right]^{-1 /(n+2)}$.

Next we extend the Legendre transform to nonsmooth (locally) convex functions.

Let $\Omega$ be a bounded $C^{2}$ smooth domain in $\mathbf{R}^{n}$ and let $\phi$ be a (locally) uniformly convex function in $\bar{\Omega}$. As before we denote by $S[\phi, \Omega]$ the set of locally uniformly convex functions $u \in C^{2}(\Omega) \cap C^{0,1}(\bar{\Omega})$ satisfying $u=\phi$ and $D u(\Omega) \subset D \phi(\Omega)$, and we denote by $\bar{S}[\phi, \Omega]$ the closure of $S[\phi, \Omega]$ under local uniform convergence. In the following we extend the Legendre transform to all functions in $\bar{S}[\phi, \Omega]$ and show that if $u$ is a maximizer for $\sup _{v \in \bar{S}[\phi, \Omega]} A(v)$, the Legendre transform of $u$ is a maximizer for $\sup _{v \in \bar{S}\left[\phi^{*}, \Omega^{*}\right]} A^{*}(v)$, where $\Omega^{*}=D \phi(\Omega)$.

First we consider the case when the domain $\Omega$ is convex such that $D \phi$ is a diffeomorphism from $\bar{\Omega}$ to $D \phi(\bar{\Omega})$. Extend $\phi$ to a neighborhood of $\bar{\Omega}$, say the domain $\Omega^{\sigma}=\left\{x \in \mathbf{R}^{n} \mid \operatorname{dist}(x, \Omega)<\sigma\right\}$ for some $\sigma>0$, such that $\phi$ is uniformly convex in $\Omega^{\sigma}$ (see [22]). For any $u \in \bar{S}[\phi, \Omega]$, we extend $u$ to $\Omega^{\sigma}$ such that $u=\phi$ in $\Omega^{\sigma}-\Omega$. Let

$$
u^{*}(y)=\sup _{x \in \Omega^{\sigma}}(x \cdot y-u(x)) \quad \forall y \in D \phi\left(\Omega^{\sigma}\right),
$$

where the supremum is attained at a point $x$ such that $y \in N_{u}(x)$. It is easy to see that $u^{*}=\phi^{*}$ in $D \phi\left(\Omega^{\sigma}-\Omega\right)$. We define the Legendre transform of $u$ as the restriction of $u^{*}$ on the domain $\Omega^{*}=D \phi(\Omega)$.

Let $\bar{S}\left[\phi^{*}\right]=\bar{S}\left[\phi^{*}, \Omega^{*}\right]$ denote the set of convex functions $v$ such that $v=\phi^{*}$ on $\partial \Omega^{*}$ and $N_{v}\left(\Omega^{*}\right) \subset \bar{\Omega}$. For any convex function $v \in \bar{S}\left[\phi^{*}, \Omega^{*}\right]$, we extend $v$ to $D \phi\left(\Omega^{\sigma}\right)$ such that $v=\phi^{*}$ in $D \phi\left(\Omega^{\sigma}-\Omega\right)$. Then similarly we can define the Legendre transform of $v$, which is a convex function on $\Omega$. 
Remark 7.1. When $\Omega$ is convex and $\phi$ is a convex function defined in $\Omega^{\sigma}$, the Legendre transform (7.5) is well defined for any $u \in \bar{S}[\phi, \Omega]$. The smoothness of $\partial \Omega$ and $\phi$ is not required. This implies that if $u$ is a convex function in a nonconvex domain $\Omega$ and $D u(x)=D u(y)$ for two points $x, y \in \Omega$ such that the line segment $\overline{x y}$ is contained in $\Omega$, then the tangent planes of $u$ at $x$ and $y$ coincide.

We remark that for any function $u \in S[\phi, \Omega]$, the usual Legendre transform of $u$ is defined by

$$
\hat{u}^{*}(y)=\sup _{x \in \Omega^{\sigma}}(x \cdot y-u(x)) \quad \forall y \in D u(\Omega) .
$$

The function $\hat{u}^{*}$ is a convex function defined in $D u(\Omega) \subset \Omega^{*}$. Obviously we have $u^{*}=\hat{u}^{*}$ in $D u(\Omega)$. The graph of $u^{*}$ in $\Omega^{*}-D u(\Omega)$ consists of line segments. In fact we have

$$
u^{*}(x)=\sup \ell(x)
$$

where the supremum is taken over all linear function satisfying $\ell \leq \hat{u}$ in $D u(\Omega)$ and $\ell \leq \phi^{*}$ on $\partial \Omega^{*}$. Our definition of the Legendre transform is an extension of the usual one such that for any function $u \in \bar{S}[\phi, \Omega], u^{*}$ is properly defined in the whole $\Omega^{*}$.

Next we consider the case when $\Omega$ is a general bounded smooth domain. In this case the gradient mapping $D \phi$ may not be one to one. We need to regard $D \phi$ as an immersion and the domain $\Omega^{*}=D \phi(\Omega)$ as an immersed manifold in $\mathbf{R}^{n}$. Let $S^{0}[\phi, \Omega]$ denote the set of uniformly convex functions $u \in S[\phi, \Omega] \cap C^{2}(\bar{\Omega})$ satisfying $u=\phi$ on $\partial \Omega$ and $D u(\Omega)=D \phi(\Omega)$. For any $u \in S^{0}[\phi, \Omega]$, extend $u$ to $\Omega^{\sigma}$ such that $u=\phi$ outside $\Omega$. Then $u$ has continuous gradient across $\partial \Omega$. It is easy to see that $\bar{S}[\phi, \Omega]$ is also the closure of $S^{0}[\phi, \Omega]$ under uniform convergence.

We claim that for any $u_{0}, u_{1} \in S^{0}[\phi, \Omega]$, there exists a diffeomorphism $\rho$ from $\Omega$ into itself, such that $D u_{0}(x)=D u_{1}(\rho(x))$. Indeed, for any $t \in[0,1]$, the function $u_{t}:=t u_{1}+(1-t) u_{0} \in S^{0}[\phi, \Omega]$. Since $u_{0}$ and $u_{1}$ are uniformly convex, namely, they are $C^{2}$ up to boundary and $D^{2} u_{0}$ and $D^{2} u_{1}$ are positive definite, there exists a diffeomorphism $\rho_{t}$ from $\Omega$ into itself, such that $D u_{t}(x)=D u_{t^{\prime}}\left(\rho_{t}(x)\right)$ if $t<t^{\prime}$ and $t, t^{\prime}$ are sufficiently close. Hence the claim follows.

It follows that $\Omega^{*}$, as a manifold immersed in $\mathbf{R}^{n}$, is also the image of the immersion $D u: \Omega \rightarrow \mathbf{R}^{n}$ for any $u \in S^{0}[\phi, \Omega]$. For $u \in S^{0}[\phi, \Omega]$, the Legendre transform of $u, u^{*}$ is a single valued function defined on the manifold $\Omega^{*}$, such that for any $y \in \Omega^{*}$,

$$
u^{*}(y)=x \cdot y-u(x),
$$

where $x \in \Omega$ is the unique point such that $y=D u(x)$. Obviously (7.6) is an extension of (7.1).

Remark 7.2. We need to explain how $u^{*}$ is understood as a single valued function on $\Omega^{*}$. Let us introduce a manifold $\mathcal{N}=\{(x, y) \mid x \in \Omega, y=D u(x)\}$ with metric $d s^{2}=d y^{2}$. Then the gradient mapping $D u$ is an embedding of $\Omega$ to $\mathcal{N}$ and $D u(\Omega)$ is isometric to $\mathcal{N}$. The function $u^{*}$ can be regarded as a single valued function on $\mathcal{N}$.

Next we define the Legendre transform for (nonsmooth) functions $u \in \bar{S}[\phi, \Omega]$. Let $\left\{u_{k}\right\} \subset S^{0}[\phi, \Omega]$ be a sequence of smooth, uniformly convex functions converging to $u$. We define $u^{*}$, the Legendre transform of $u$, by

$$
u^{*}(y)=\lim _{k \rightarrow \infty} u_{k}^{*}(y)
$$


where $u_{k}^{*}$ is the Legendre transform of $u_{k}$, given in (7.6). To show that $u^{*}$ is well defined, we need to show that $u^{*}$ is independent of the choice of the sequences. Suppose $\left\{u_{k}^{1}\right\} \subset S^{0}[\phi, \Omega]$ is another sequence of smooth, uniformly convex functions which converges to $u$. For any point $y$ on the manifold $\Omega^{*}$, there is a unique point $x_{k} \in \Omega\left(x_{k}^{1} \in \Omega\right.$, resp. $)$ such that $D u_{k}\left(x_{k}\right)=y\left(D u_{k}^{1}\left(x_{k}^{1}\right)=y\right.$, resp.). By choosing subsequences, we suppose $x_{k} \rightarrow x_{0}$ and $x_{k}^{1} \rightarrow x_{0}^{1}$.

We claim that there exists a curve $\ell \subset \bar{\Omega}$ connecting $x_{0}$ and $x_{0}^{1}$ such that $y \in$ $N_{u}(x)$ for any $x \in \ell$. Suppose for a moment the claim is true. Since $u$ can be extended to a convex function in a neighborhood of $\Omega$ (namely, $u=\phi$ on $\Omega^{\sigma}-\Omega$ ), we can divide $\ell$ into the union of small arcs, $\ell=\bigcup_{i} \ell_{i}$, such that for each $\ell_{i}$, the line segment $\hat{\ell}_{i}$ connecting the two endpoints of $\ell_{i}$ lies in $\Omega^{\sigma}$. Let $x_{0}, \cdots, x_{m}$ be the endpoints of these line segments such that $\hat{\ell}_{i}=\overline{x_{i} x_{i+1}}$. Since $y \in N_{u}\left(x_{i}\right)$, the hyperplane $P_{i}=\left\{\left(x, x_{n+1}\right) \in \mathbf{R}^{n+1} \mid x_{n+1}=y \cdot\left(x-x_{i}\right)+u\left(x_{i}\right)\right\}$ is a tangent plane of $u$ at $x_{i}$. By convexity $u$ is linear on these line segments $\hat{\ell}_{i}$ and the tangent planes of $u$ at $x_{i}$ and $x_{i+1}$ coincide; see Remark 7.1. Therefore we obtain $x_{0} \cdot y-u\left(x_{0}\right)=$ $x_{0}^{1} \cdot y-u\left(x_{0}^{1}\right)$, namely, the limit in (7.7) does not depend on the choice of the sequence $\left\{u_{k}\right\}$. By Remark 7.1, one also sees that the Legendre transform depends locally on $u$.

To prove the claim, we let $u_{k}^{t}=t u_{k}+(1-t) u_{k}^{1}$. Then for any $t \in[0,1]$, $\left\{u_{k}^{t}\right\} \subset S^{0}[\phi, \Omega]$ and $u_{k}^{t} \rightarrow u$ as $k \rightarrow \infty$. Let $x_{k}^{t}$ be the unique point in $\Omega$ such that $D u_{k}^{t}\left(x_{k}^{t}\right)=y$. By the uniform convexity of $u_{k}$ and $u_{k}^{1}, x_{k}^{t}$ depends continuously on $t$. Hence for fixed $k, E_{k}=\left\{x_{k}^{t} \mid t \in[0,1]\right\}$ is a curve in $\Omega$. Let $\mathcal{E}$ denote the set of such points $\hat{x}$ for which there is a sequence $\left\{\hat{x}_{k}\right\}$, where $\hat{x}_{k} \in E_{k}$, which sub-converges to $\hat{x}$. For any given $\hat{x} \in \mathcal{E}$, since the hyperplane $P_{k}=\left\{x_{n+1}=\right.$ $\left.y \cdot\left(x-x_{k}^{t}\right)+u_{k}^{t}\left(x_{k}^{t}\right)\right\}$ is a tangent plane of $u_{k}^{t}$ at $x_{k}^{t}$ and $u_{k}^{t}$ converges to $u$ uniformly, $\mathcal{P}=\left\{x_{n+1}=y \cdot(x-\hat{x})+u(\hat{x})\right\}$ is a tangent plane of $u$ at $\hat{x}$. It follows that $y \in N_{u}(\hat{x})$ for any $\hat{x} \in \mathcal{E}$. Since $E_{k}$ is connected, for any $r>0$ small such that $B_{r}(\hat{x}) \subset \Omega^{\sigma}$ and $E_{k} \cap \partial B_{r}(\hat{x}) \neq \emptyset$, there is a point $\hat{x}_{r} \in \partial B_{r}(\hat{x}) \cap \mathcal{E}$. Namely, $\mathcal{P}_{r}=\left\{x_{n+1}=y \cdot\left(x-\hat{x}_{r}\right)+u\left(\hat{x}_{r}\right)\right\}$ is a tangent plane of $u$ at $\hat{x}_{r}$. When $r$ is sufficiently small, by convexity we see that $\mathcal{P}$ and $\mathcal{P}_{r}$ must coincide. Again by the convexity of $u$, we see that $u$ is linear on the line segment $\bar{x} \hat{x}_{r}$ and so $y \in N_{u}(x)$ for any $x$ on the line segment. The claim is proved. Hence (7.7) is well defined.

Regarding $\Omega^{*}$ as a manifold (see Remark 7.2), we can define the Legendre transform for functions $v \in \bar{S}\left[\phi^{*}, \Omega^{*}\right]$ similarly. Indeed, note that $S^{0}\left[\phi^{*}, \Omega^{*}\right]$ is the set of Legendre transforms of functions in $S^{0}[\phi, \Omega]$; see (7.6). Hence if $v \in S^{0}\left[\phi^{*}, \Omega^{*}\right]$, we can define the Legendre transform for a function $v \in S^{0}\left[\phi^{*}, \Omega^{*}\right]$ by

$$
v^{*}(x)=x \cdot y-v(y),
$$

where $y \in \Omega^{*}$ is the unique point such that $x=D v(y)$. Obviously $v^{*} \in S^{0}[\phi, \Omega]$ and $v$ is the Legendre transform of $v^{*}$. For general functions $v \in \bar{S}\left[\phi^{*}, \Omega^{*}\right]$, the Legendre transform of $v$ is also defined as the limit of the Legendre transforms of sequences of smooth, locally uniformly convex functions in $S^{0}\left[\phi^{*}, \Omega^{*}\right]$ which converge to $v$. From (7.7), this is well defined.

Remark 7.3. It is easy to see that if $\left\{u_{k}\right\}$ is a sequence in $\bar{S}[\phi, \Omega]$ and $u_{k} \rightarrow u$ uniformly, then the Legendre transform $u_{k}^{*}$ converges to $u^{*}$. Observe that $u \in$ $S^{0}[\phi, \Omega]$ if and only if $u^{*} \in S^{0}\left[\phi^{*}, \Omega^{*}\right]$. Hence for any $u \in \bar{S}[\phi, \Omega]$, we have $\left(u^{*}\right)^{*}=u$, namely, the Legendre transform of $u^{*}$ is $u$ itself. 
Lemma 7.1. Let $u \in \bar{S}[\phi, \Omega]$ and let $u^{*}$ be its Legendre transform. Then

$$
A(u, \Omega)=A^{*}\left(u^{*}, \Omega^{*}\right),
$$

where $\Omega^{*}$ is regarded as a manifold, as noted in Remark 7.2.

Proof. If $u$ is twice differentiable at $x$ and $\operatorname{det} \partial^{2} u(x)>0$, then it is easy to prove that $u^{*}$ is twice differentiable at $y=D u(x)$, and by (7.2) one has the relation

$$
\left(\partial^{2} u^{*}(y)\right)\left(\partial^{2} u(x)\right)=I,
$$

where $I$ is the unit matrix.

By our definition of the Legendre transform, (7.9) is obviously true for uniformly convex functions in $S^{0}[\phi, \Omega]$. We need to prove (7.9) for general convex functions in $\bar{S}[\phi, \Omega]$. For any Borel set $E \subset \Omega$ with $\operatorname{dist}(E, \partial \Omega) \geq \delta$, and any $\alpha \in(0,1)$, we have

$$
\begin{aligned}
\int_{E}\left(\operatorname{det} \partial^{2} u\right)^{\alpha} & \leq|E|^{1-\alpha}\left(\int_{E} \operatorname{det} \partial^{2} u\right)^{\alpha} \\
& =|E|^{1-\alpha}\left(\mu_{r}[u](E)\right)^{\alpha} \\
& \leq|E|^{1-\alpha}(\mu[u](E))^{\alpha} \\
& \leq|E|^{1-\alpha}\left(\frac{\operatorname{osc} u}{\delta}\right)^{n \alpha}
\end{aligned}
$$

where $\mu_{r}[u]$ is the regular part of the Monge-Ampère measure $\mu[u]$. It follows that $\left(\operatorname{det} \partial^{2} u\right)^{\alpha}$ is locally equi-integrable whenever $u$ is bounded.

Extend $u$ to $\Omega^{\sigma}$ such that $u=\phi$ outside $\Omega$. Let $u_{h}$ be the mollification of $u$, as defined in (2.3). Then $D^{2} u_{h}(x) \rightarrow \partial^{2} u(x)$ whenever $u$ is twice differentiable at $x$ [25]. Let $\hat{u}_{h}=u_{h}+h|x|^{2}$. Then we have

$$
\int_{\Omega}\left(\operatorname{det} \partial^{2} u\right)^{\alpha}=\lim _{h \rightarrow 0} \int_{\Omega}\left(\operatorname{det} D^{2} \hat{u}_{h}\right)^{\alpha} .
$$

Let $\hat{u}_{h}^{*}$ be the Legendre transformation of $\hat{u}_{h}$. By $(7.10)$ we have $D^{2} \hat{u}_{h}^{*}(y) \rightarrow \partial^{2} u^{*}(y)$ whenever $u^{*}$ is twice differentiable at $y$ and $\operatorname{det} \partial^{2} u^{*}(y)>0$. It follows that

$$
\int_{\Omega^{*}}\left(\operatorname{det} \partial^{2} u^{*}\right)^{\alpha} \leq \lim _{h \rightarrow 0} \int_{\Omega^{*}}\left(\operatorname{det} D^{2} \hat{u}_{h}^{*}\right)^{\alpha} .
$$

Since (7.9) holds for uniformly convex functions in $S^{0}[\phi, \Omega]$, we obtain

$$
A^{*}\left(u^{*}, \Omega^{*}\right) \leq A(u, \Omega) .
$$

For any $v \in \bar{S}\left[\phi^{*}, \Omega^{*}\right]$, let $v^{*}$ be the Legendre transform of $v$. Then similarly we have

$$
A\left(v^{*}, \Omega\right) \leq A^{*}\left(v, \Omega^{*}\right) .
$$

Observe that the Legendre transform of $u^{*}$ is $u$ itself. Choosing $v=u^{*}$ such that $v^{*}=u$, we obtain (7.9).

Similarly to Theorem 2.1, the functional $A^{*}$ is upper semi-continuous, so there exists a maximizer $v$ to the supremum

$$
\sup _{v \in \bar{S}\left[\phi^{*}, \Omega^{*}\right]} A^{*}(v) .
$$

If $v$ is smooth and uniformly convex, it satisfies the Euler equation (7.4) with $f=0$. 
Let $u$ be the Legendre transform of $v$. From (7.9) we see that $u$ is the maximizer for $\sup _{u \in \bar{S}[\phi, \Omega]} A(u)$, and

$$
\sup _{u \in \bar{S}[\phi, \Omega]} A(u)=\sup _{v \in \bar{S}\left[\phi^{*}, \Omega^{*}\right]} A^{*}(v) .
$$

Furthermore, $v$ is the Legendre transform of $u$, and hence by Theorem 2.1, $v$ is unique.

For locally convex hypersurfaces, the notion of support function plays a similar role to the Legendre transform for convex graphs. Since a locally convex hypersurface can be decomposed as a union of graphs (see the proof of Theorem 3.1), we will just discuss support functions for convex hypersurfaces, that is, hypersurfaces lying in the boundaries of convex bodies.

Let $\mathcal{M}$ be a smooth, convex hypersurface with Gauss mapping image $\mathcal{N}$. The support function $H$ is a function defined on $\mathcal{N}$, given by

$$
H(x)=\sup \{p \cdot x \mid p \in \mathcal{M}\} .
$$

If $\mathcal{M}$ is locally uniformly convex, the supremum is attained at the unique point $p \in \mathcal{M}$ with unit outward normal $x$. Moreover, the Gauss curvature of $\mathcal{M}$ at $p$ is given by

$$
K=1 / \operatorname{det}\left(\nabla^{2} H+H I\right)(x),
$$

where $\nabla$ is the covariant derivative on $S^{n}$ (under a local orthonormal frame), and $I$ is the unit matrix. Extend $H$ to $\mathbf{R}^{n+1}$ such that it is homogeneous of degree one, namely, $H(t x)=t H(x)$ for any $t \geq 0$ and $x \in \mathcal{N}$. Then $\mathcal{M}$ can be recovered from $H$ by

$$
\mathcal{M}=\left\{D H(x) \in \mathbf{R}^{n+1}: \mid x \in \mathcal{N}\right\},
$$

where $D$ is the ordinary derivative in $\mathbf{R}^{n+1}$. If locally $\mathcal{M}$ is represented as a graph of a convex function $u$, then

$$
u^{*}(y)=H(y,-1), \quad y \in \Omega^{*},
$$

is exactly the Legendre transform of $u$. By (7.16) we have

$$
\operatorname{det} D^{2} u^{*}(y)=\left(1+|y|^{2}\right)^{-(n+2) / 2} \operatorname{det}\left(\nabla^{2} H+H I\right) .
$$

Note that in the graph case, (7.17) is equivalent to the Legendre transform for $u^{*}$.

For an arbitrary convex hypersurface $\mathcal{M}$, one can define the support function using the generalized Gauss mapping. For any interior point $p \in \mathcal{M}$, the generalized Gauss mapping is a multi-valued mapping given by

$$
G(p)=\left\{x \in S^{n} \mid x \cdot \widetilde{p} \leq x \cdot p \quad \forall \widetilde{p} \in \mathcal{M}\right\} .
$$

Let $\mathcal{N}=\bigcup G(p)$, where the union is taken over all interior points of $\mathcal{M}$. Then we can define the support function of $\mathcal{M}$ also by (7.15), and if $\mathcal{M}$ is a graph, the support function is related to the Legendre transform by (7.18).

\section{StRict CONVEXITY}

Throughout this section we assume the dimension $n=2$. In two dimensions, the local strict convexity of affine maximal functions which vanish on the boundary of a convex domain $\Omega$ follows from our treatment of the affine Bernstein problem in [21] and the approximation in Section 6. 
Theorem 8.1. Suppose that $u \in C^{0}(\bar{\Omega})$ is an affine maximal convex function in a domain $\Omega \subset \mathbf{R}^{2}$, vanishing on the boundary $\partial \Omega$. Then $u$ is strictly convex in $\Omega$ and the modulus of convexity of $u$ can be estimated in term of $\Omega$ and $\inf _{\Omega} u$.

We remark that Theorem 8.1 holds for affine maximal graphs with boundary lying on a plane. Using the affine invariance of the affine maximal surface equation, the proof of Theorem 8.1 reduces to the fact that a convex function with bounded Monge-Ampère measure is differentiable at any point on its graph, not lying on a line segment joining two boundary points. This is embodied in the following lemma.

Lemma 8.1. Suppose $u$ is a nonnegative convex function in a domain $\Omega \ni\{0\}$, $\subset \mathbf{R}^{2}$, satisfying $u>0$ on $\partial \Omega, u(0)=0$ and $u\left(x_{1}, 0\right) \geq\left|x_{1}\right|$. Then the MongeAmpère measure $\mu[u]$ cannot be a bounded function.

Proof. We outline the proof here, since it is essentially contained in 21], Section 5. First by Lemma 2.3, the Radon-Nikodym derivative of $\mu[u]$ is an integrable function. If the set $\{u=0\}$ is the single point $\{0\}$, let $G_{\varepsilon}=\{u<\varepsilon\}$. Then the Lebesgue measure $\left|G_{\varepsilon}\right| \leq \varepsilon \delta_{\varepsilon}$, where $\delta_{\varepsilon}=\sup \left\{\left|x_{2}\right| \mid\left(x_{1}, x_{2}\right) \in G_{\varepsilon}\right\}$. On the other hand, the image of the normal mapping $N_{u}\left(G_{\varepsilon}\right)$ satisfies $\left|N_{u}\left(G_{\varepsilon}\right)\right|>C \varepsilon / \delta_{\varepsilon}$ for some $C>0$ independent of $\varepsilon$. By definition we have $\mu[u]\left(G_{\varepsilon}\right)=\left|N_{u}\left(G_{\varepsilon}\right)\right|$. Hence $\mu[u]$ is unbounded near the origin.

If the set $\{u=0\}$ is a line segment, by the assumption that $u>0$ on $\partial \Omega$, we may suppose $\{u=0\}$ is the line segment $\left\{(0,-t) \mid 0 \leq t \leq t_{0}\right\}$ such that the origin is an endpoint. Let $G_{\varepsilon}=\left\{u<\ell_{\varepsilon}\right\}$, where $\ell_{\varepsilon}(x)=\varepsilon+\delta_{\varepsilon} x_{2}$ is a linear function, and $\delta_{\varepsilon}>0$ is chosen such that $u\left(0, \varepsilon / \delta_{\varepsilon}\right)=\ell_{\varepsilon}\left(0, \varepsilon / \delta_{\varepsilon}\right)=2 \varepsilon$ and $u\left(0,-\varepsilon / \delta_{\varepsilon}\right)=$ $\ell_{\varepsilon}\left(0,-\varepsilon / \delta_{\varepsilon}\right)=0$. By the convexity and the assumption that $u\left(x_{1}, 0\right) \geq\left|x_{1}\right|$, we see that $G_{\varepsilon} \subset\left\{-\delta_{\varepsilon} \leq x_{2} \leq C \delta_{\varepsilon}\right\}$ for some $C>0$ depending on sup $|D u|$. Similarly as above we have $\left|G_{\varepsilon}\right| \leq C \varepsilon \delta_{\varepsilon}$, and $\left|N_{u}\left(G_{\varepsilon}\right)\right|>C \varepsilon / \delta_{\varepsilon}$ for a different $C>0$. Hence $\mu[u]$ is unbounded near the origin.

We say a point $x_{0} \in \partial F$ is an extreme point of a convex set $F \subset \mathbf{R}^{n}$ if there is a hyperplane $P$ such that intersection $P \cap \bar{F}$ is the single point $\left\{x_{0}\right\}$. The main result of this section is the following strict convexity in dimension 2 for affine maximal graphs with general boundary values.

Theorem 8.2. Let $\Omega$ be a bounded $C^{2}$ smooth domain in $\mathbf{R}^{2}$ and let $\phi$ be a uniformly convex function in $\bar{\Omega}$. Then the maximizer $u$ in Theorem 2.1 is strictly convex in $\Omega$, in the case of vanishing $f$.

Proof. If $u$ is not strictly convex, then the graph of $u, \mathcal{M}_{u}$, contains a line segment $\ell$. Let $P$ be the tangent plane of $\mathcal{M}_{u}$ containing $\ell$. Let $F$ be the component of $P \cap \mathcal{M}_{u}$ which contains $\ell$ (note that $P \cap \mathcal{M}_{u}$ may contain more than one component if $\Omega$ is not convex). Then $F$ is a convex set. We have two cases.

Case (a). $F$ has an extreme point $x_{0}$ which is an interior point of $\mathcal{M}_{u}$. Then there is a plane $P_{0}=\{v(x)=a \cdot x+b\}$, such that $v\left(x_{0}\right)>u\left(x_{0}\right)$ and $v<u$ on $\partial \Omega$. Let $\Omega^{\prime}=\{x \in \Omega \mid v(x)>u(x)\}$. Then $u$ is strictly convex in $\Omega^{\prime}$ by Theorem 8.1. This is a contradiction since $x_{0}$ is an extreme point of $F$.

Case (b). All extreme points of $F$ are boundary points of $\Omega$. In this case we suppose for simplicity that $P=\left\{x_{3}=0\right\}$ and $u \geq 0$ near $F$. Since all extreme points of $F$ are boundary points of $\Omega$, there exists a line segment contained in $F$, which we suppose is

$$
\ell=\left\{t e_{1} \mid-1 \leq t \leq 1\right\},
$$


where $e_{1}=(1,0)$, such that both endpoints of $\ell$ are boundary points of $\mathcal{M}_{u}$. By the uniform convexity of $\phi$ we see that $\ell$ is transversal to $\partial \Omega$ at the endpoints $\pm e_{1}$, namely, $\left|\left\langle e_{1}, \gamma\right\rangle\right| \geq C>0$, for otherwise we have $\frac{\partial^{2}}{\partial x_{1}^{2}} \phi=0$ at the endpoints of $\ell$, where $\gamma$ is the unit outward normal to $\partial \Omega$. Since $\phi=u \geq 0$ on $\partial \Omega$ and $u=0$ at the endpoints $\pm e_{1}$, by the smoothness of $\phi$ and $\partial \Omega$, we have $|u(x)| \leq \frac{1}{2} C x_{2}^{2}$ for $x \in \partial \Omega$, close to the endpoints $\pm e_{1}$. By convexity it follows that

$$
u(x) \leq \frac{C}{2} x_{2}^{2}
$$

for $x \in \Omega$, close to the segment $\ell$.

Let $u^{*}$ be the Legendre transform of $u$, as introduced in Section 7. Then $u^{*}$ is the maximizer of the functional $A^{*}$ over the set $\bar{S}\left[\phi^{*}, \Omega^{*}\right]$ (see (7.14)), and the origin is an interior point of $\Omega^{*}$, where $\Omega^{*}=D \phi(\Omega)$. By (8.2) and since $u \geq 0$, we have $u^{*}(0)=0$ and

$$
u^{*}\left(x_{1}, x_{2}\right) \geq \frac{1}{2 C} x_{2}^{2}
$$

near the origin. Since the line segment $\ell$ in (8.1) is contained in $F$, we have

$$
u^{*}\left(x_{1}, x_{2}\right) \geq\left|x_{1}\right|
$$

locally near the origin. In the following we prove that the function $u^{*}$, which satisfies (8.3) and (8.4), cannot be a maximizer of $A^{*}$ over the set $\bar{S}\left[\phi^{*}, \Omega^{*}\right]$. Before continuing our proof, we make the following remarks.

Remark 8.1. By Lemma 8.1 the Radon-Nikodym derivative of the Monge-Ampère measure $\mu\left[u^{*}\right]$ is an unbounded function. On the other hand if one can prove that $u^{*}$ can be approximated by smooth solutions of (7.4) (with $f=0$ ), then $\mu\left[u^{*}\right]$ is bounded by Lemma 4.1 or Remark 4.1. However at the moment we do not know if $u^{*}$ can be approximated by smooth solutions since our proof of the approximation result in Section 6 depended on the concavity of the affine area functional $A$; see (5.10) and (6.13).

Remark 8.2. Case (b) can occur if $\phi$ is not uniformly convex. Indeed, if the graph of $\phi$ contains a line segment of which both endpoints are boundary points, then the line segment lies on the graph of any function in $\bar{S}[\phi]$.

Remark 8.3. In Section 7 the Legendre transform of $u$ is defined on $D \phi(\Omega)$, which is regarded as an immersed manifold in $\mathbf{R}^{n}$. Here in the proof of Theorem 8.2, we need only to consider the piece of $\mathcal{M}_{u, \delta}=\left\{(x, u(x)) \in \mathcal{M}_{u} \mid u(x)<\delta\right\}$ for small $\delta>0$, where (as in Section 7) $u$ is extended to a neighborhood of $\Omega$ such that $u=\phi$ outside $\Omega$. The Legendre transform of $u$ introduced in Section 7, when restricted to the piece $\mathcal{M}_{u, \delta}$, coincides with

$$
u^{*}(y)=\sup _{x \in \omega}(x \cdot y-u(x)) \quad \forall y \in \omega^{*}=N_{u}(\omega)
$$

since $\omega$ is convex, where $\omega=\omega_{\delta}$ is the projection of $\mathcal{M}_{u, \delta}$ on $\left\{x_{n+1}=0\right\}$. Moreover, $u$ can be recovered from $u^{*}$ by the same formula, that is,

$$
u(x)=\sup _{y \in \omega^{*}}\left(x \cdot y-u^{*}(y)\right) \quad \forall x \in \omega .
$$

In the following we suppose directly that the Legendre transform of $u$ is given by the above formula. 
Remark 8.4. We shall use the following basic property of the Legendre transform. If $x_{0} \in \bar{\Omega}$ and $y_{0} \in N_{u}\left(x_{0}\right)$, then $x_{0} \in N_{u^{*}}\left(y_{0}\right)$ and locally $u^{*}(y) \geq x_{0} \cdot y-u\left(x_{0}\right)$, namely, $x_{0} \cdot y-u\left(x_{0}\right)$ is a support plane of $u^{*}$ at $y_{0}$, where $N_{u}$ is the normal mapping introduced in Section 2. Since the endpoints $\pm e_{1}$ of $\ell$ are boundary points of $\partial \Omega$, we have $\lim _{x_{1} \rightarrow 0} \frac{1}{\left|x_{1}\right|} u^{*}\left(x_{1}, 0\right)=1$.

Returning to the proof of Theorem 8.2, we denote $g(t)=u^{*}(0, t)$. Then $g(0)=0$ and by $(8.3)$

$$
g(t) \geq t^{2}
$$

Note that we may suppose $C \leq 1 / 2$ in (8.3) by replacing $u$ by $u / 2 C$ in (8.2). Denote by $\Omega_{h}=\left\{x \in \mathbf{R}^{2} \mid u^{*}(x)<h\right\}$ the sub-level set of $u^{*}$, where $h>0$. We also denote by $t_{h}^{+}>0$ and $t_{h}^{-}<0$ the unique constants satisfying $g\left(t_{h}^{ \pm}\right)=h$. First we show that $\Omega_{h}$ has "good" shape.

Lemma 8.2. There is a positive constant $C>0$, independent of $h$, such that

$$
\Omega_{h} \subset\left\{x \in \mathbf{R}^{2} \mid-h \leq x_{1} \leq h, C t_{h}^{-} \leq x_{2} \leq C t_{h}^{+}\right\} .
$$

Proof. By (8.4) we have $\Omega_{h} \subset\left\{-h \leq x_{1} \leq h\right\}$. We need only to prove $\Omega_{h} \subset$ $\left\{C t_{h}^{-} \leq x_{2} \leq C t_{h}^{+}\right\}$. By (8.4), this is true with $C=2$ if $u^{*}\left(x_{1}, 0\right)=\left|x_{1}\right|$. In general we will prove $\Omega_{h} \subset\left\{x_{2} \leq C t_{h}^{+}\right\}$by restricting ourselves to the region $\Omega_{h} \cap\left\{x_{2} \geq 0\right\}$. For $h>0$ small, let

$$
u_{h}^{*}\left(x_{1}, x_{2}\right)=h^{-1} u^{*}\left(h x_{1}, t_{h}^{+} x_{2}\right) .
$$

Then the graph of $u_{h}^{*}$ (the part in $\left\{x_{3}<1\right\}$ ) sub-converges, as $h \rightarrow 0$, to a convex surface $\mathcal{M}_{0}^{*}$ such that the point $(0,1,1) \in \partial \mathcal{M}_{0}^{*}$. Since the endpoints of $\ell$ are boundary points of $\Omega$, we have $\lim _{x_{1} \rightarrow 0} \frac{1}{\left|x_{1}\right|} u^{*}\left(x_{1}, 0\right)=1$. Hence the line segments $\left\{x_{3}=\left|x_{1}\right|, x_{1} \in(-1,1), x_{2}=0\right\}$ lie on $\mathcal{M}_{0}^{*}$. On the other hand, since $u^{*}\left(x_{1}, x_{2}\right) \geq$ $\left|x_{1}\right|$, we see that $\mathcal{M}_{0}^{*}$ lies above the planes $x_{3}= \pm x_{1}$. By convexity it follows that $\mathcal{M}_{0}^{*} \subset\left\{y_{2}<2\right\}$. Hence $\Omega_{h} \subset\left\{x_{2} \leq C t_{h}^{+}\right\}$with $C \rightarrow 2$ as $h \rightarrow 0$. Similarly one can prove $\Omega_{h} \subset\left\{x_{2} \geq C t_{h}^{-}\right\}$with $C \rightarrow 2$ as $h \rightarrow 0$.

In the above proof, a sequence of convex surfaces $\left\{\Gamma_{k}\right\}$ is said to converge to a surface $\Gamma_{\infty}$ if for any given $R>0$ and any $\delta>0$, there exists $k>1$ such that for any $j \geq k, \Gamma_{j} \cap B_{R}(0) \subset N_{\delta}\left(\Gamma_{\infty} \cap B_{R}\right)$ and $\Gamma_{\infty} \cap B_{R}(0) \subset N_{\delta}\left(\Gamma_{j} \cap B_{R}\right)$, where $N_{\delta}$ denotes the $\delta$-neighborhood.

By the proof of Lemma 2.3, the singular part of the Monge-Ampère measure $\mu[u]$ vanishes. Hence

$$
\lim _{t \rightarrow 0} g(t) /|t|=0
$$

Lemma 8.3. There is a constant $\beta>0$ such that

$$
g(t) \leq|t|^{1+\beta}
$$

for small $t$.

Proof. Denote $\bar{g}(t)=g(t)+g(-t)$, where $t>0$. It suffices to prove that $\bar{g}$ satisfies (8.7). We need only to prove that there is a positive constant $\theta>0$, independent of $h$ such that

$$
\bar{g}\left(\frac{1}{2} t\right) \leq\left(\frac{1}{2}-\theta\right) \bar{g}(t)
$$

If (8.8) is not true, there exists a sequence $t_{j} \rightarrow 0$ such that $\bar{g}\left(\frac{1}{2} t_{j}\right) \geq\left(\frac{1}{2}-2^{-j}\right) \bar{g}\left(t_{j}\right)$. Let $\bar{g}_{j}(t)=a_{j}^{-1} \bar{g}\left(t_{j} t\right)$ and let $u_{j}^{*}\left(x_{1}, x_{2}\right)=a_{j}^{-1} u^{*}\left(a_{j} x_{1}, t_{j} x_{2}\right)$, where $a_{j}=\bar{g}\left(t_{j}\right)$. 
Then $u_{j}^{*}$ sub-converges to a convex function $u_{0}^{*}$, and $\bar{g}_{j}$ converges to a linear function $\bar{g}_{0}$ and $\bar{g}_{0}(t)=u_{0}^{*}(0, t)+u_{0}^{*}(0,-t)$. By convexity it follows that $u_{0}^{*}(0, t)$ is linear in both $t>0$ and $t<0$. On the other hand, since $\lim _{x_{1} \rightarrow 0} \frac{1}{\left|x_{1}\right|} u^{*}\left(x_{1}, 0\right)=1$, we have $u_{0}^{*}\left(x_{1}, 0\right)=\left|x_{1}\right|$. Hence $\left|N_{u_{0}^{*}}(\{0\})\right|>0$, that is, the singular part of the MongeAmpère measure $\mu\left[u_{0}^{*}\right]$ does not vanish at the origin. On the other hand, since $u^{*}$ is a maximizer (see (7.14)), $u_{0}^{*}$ is also a maximizer, and so the singular part of $\mu\left[u_{0}^{*}\right]$ must vanish by the proof of Lemma 2.3. We reach a contradiction.

Let $\alpha^{ \pm}=\alpha_{h}^{ \pm}$be the constants such that

$$
\left[t_{h}^{+}\right]^{1+\alpha^{+}}=h, \quad\left[t_{h}^{-}\right]^{1+\alpha^{-}}=h,
$$

and let $\alpha_{h}=\min \left(\alpha^{+}, \alpha^{-}\right)$. By (8.5) and (8.7) we have

$$
\beta \leq \alpha_{h} \leq 1 \text {. }
$$

Denote $\alpha_{0}=\underline{\lim }_{h \rightarrow 0} \alpha_{h}$. Choose a sufficiently small $h>0$ such that

$$
\alpha_{h} \leq \alpha_{0}+\varepsilon,
$$

where $\varepsilon>0$ is a sufficiently small constant. Denote $\alpha=\min \left(\alpha_{h}^{+}, \alpha_{h}^{-}\right)$. Suppose without loss of generality that $\alpha=\alpha_{h}^{+}$. Denote $\Omega_{h}^{+}=\Omega_{h} \cap\left\{x_{2}>-h^{1 /(1+\alpha)}\right\}$ and $q_{h}=\left|N_{u^{*}}\left(\Omega_{h}^{+}\right)\right|$. Then by Lemma 8.2,

$$
\left|\Omega_{h}^{+}\right| \leq C h^{1+1 /(1+\alpha)} .
$$

We need a lower bound for $q_{h}$.

Lemma 8.4. For $h>0$ small, we have

$$
q_{h} \geq C h^{\alpha /(1+\alpha)}
$$

for some $C>0$ independent of $h$.

Proof. $u_{h}^{*}\left(x_{1}, x_{2}\right)=h^{-1} u^{*}\left(h x_{1}, t_{h}^{+} x_{2}\right)$, where $t_{h}^{+}=h^{1 /(1+\alpha)}$. Then (8.11) is equivalent to $\left|N_{u_{h}^{*}}\left(G_{h}\right)\right| \geq C$, where $G_{h}=\left\{x \in \mathbf{R}^{2} \mid u_{h}^{*}(x)<1, x_{2}>-1\right\}$.

Let $\omega_{h}=\left\{x \in \mathbf{R}^{2} \mid u_{h}^{*}(x)<a\left(x_{2}+1\right)\right\}$. By (8.6) we see that $\omega_{h} \subset G_{h}$ if $a>0$ is small (one can choose $a=\frac{1}{3}$ for $h>0$ small). Let $z_{h}$ be a convex function such that $z(0)=0, z(x)=u_{h}^{*}(x)$ on $\partial \omega_{h}$, and $z(t x)=t z(x)$ for any $t \geq 0$. Then the graph of $z$ is a convex cone. It is easy to see that

$$
\left|N_{u_{h}^{*}}\left(G_{h}\right)\right| \geq\left|N_{z_{h}}(\{0\})\right| \geq C .
$$

Hence (8.11) holds.

By a rescaling we suppose $B_{1}(0) \subset \Omega^{*}$. Let $v$ be the solution of

$$
\mu[v]=\left(1-\chi_{\Omega_{h}^{+}}\right) \mu\left[u^{*}\right]+\delta \chi_{\widetilde{E}}
$$

such that $v=u^{*}$ on $\partial B_{1}$, where

$$
\widetilde{E}=\Omega_{h^{*}} \cap\left\{\left|x_{2}\right|<\frac{1}{2}\left(h^{*}\right)^{1 /\left(1+\alpha^{*}\right)}\right\},
$$

$h^{*}$ is given in (8.20) below, and $\chi_{\widetilde{E}}$ is the characteristic function of $\widetilde{E}$. We choose the constant $\delta>0$ such that

$$
\delta|\widetilde{E}|=\frac{1}{2} q_{h} .
$$


We claim that there is a point $x_{0} \in \Omega_{h}^{+}$such that

$$
v\left(x_{0}\right)>u^{*}\left(x_{0}\right)+c_{0} h^{\alpha /(1+\alpha)}
$$

for some constant $c_{0}>0$ independent of $h$. Indeed, if (8.14) is not true, let

$$
\bar{v}=v+c_{0} h^{\alpha /(1+\alpha)}\left(|x|^{2}-1\right) .
$$

Then $\bar{v} \leq u^{*}$ on $\partial\left(B_{1}-\Omega_{h}^{+}\right)$and $\mu[\bar{v}] \geq \mu[v] \geq \mu\left[u^{*}\right]$ in $B_{1}-\Omega_{h}^{+}$. Hence by the comparison principle we conclude that $\bar{v} \leq u^{*}$ in $B_{1}-\Omega_{h}^{+}$. It follows that

$$
\left|N_{\bar{v}}\left(B_{1}\right)\right| \geq\left|N_{u^{*}}\left(B_{1}\right)\right| .
$$

The following lemma provides an upper bound for $\left|N_{\bar{v}}\left(B_{1}\right)\right|$.

Lemma 8.5. We have

$$
\left|N_{\bar{v}}\left(B_{1}\right)\right| \leq\left|N_{v}\left(B_{1}\right)\right|+c_{1} h^{\alpha /(1+\alpha)},
$$

where $c_{1}$ can be arbitrarily small as long as $c_{0}$ is sufficiently small.

To use Lemma 8.5, from (8.12) we have

$$
\left|N_{v}\left(B_{1}\right)\right|=\left|N_{u^{*}}\left(B_{1}-\Omega_{h}^{+}\right)\right|+\frac{1}{2} q_{h}=\left|N_{u^{*}}\left(B_{1}\right)\right|-\frac{1}{2} q_{h} .
$$

Hence when $c_{0}>0$ is chosen small, by (8.11) and (8.16) we have

$$
\left|N_{\bar{v}}\left(B_{1}\right)\right| \leq\left|N_{u^{*}}\left(B_{1}\right)\right|-\frac{1}{2} q_{h}+c_{1} h^{\alpha /(1+\alpha)}<\left|N_{u^{*}}\left(B_{1}\right)\right|,
$$

which contradicts (8.15). Hence (8.14) holds.

Proof of Lemma 8.5. By approximation we may suppose $v$ is smooth. Then $G=$ $D v\left(B_{1}\right)$ is a bounded topological disc. Let $\partial B_{1}$ be parametrized by $\theta \in[0,2 \pi]$, namely, $x=(\cos \theta, \sin \theta)$. Then $D v$ is a diffeomorphism from $\partial B_{1}$ to $\partial G$. By convexity we have

$$
\left\langle\frac{d}{d \theta} D v(x), \frac{d}{d \theta} x\right\rangle>0, \quad x \in \partial B_{1},
$$

where $\langle\cdot, \cdot\rangle$ denotes the inner product in $\mathbf{R}^{2}$. For any $x \in \partial B_{1}$, we have

$$
D \bar{v}(x)=D v(x)+b x,
$$

where $b=2 c_{0} h^{\alpha /(1+\alpha)}$. If $G$ is a ball, (8.16) follows immediately from (8.17) and (8.18), with $c_{1}=4 \pi c_{0}$.

In general we choose $R>0$ such that $G$ is contained in the ball $B_{R}$. Observe that $N_{\bar{v}}\left(B_{1}\right)-N_{v}\left(B_{1}\right)$ is the region covered by the family of line segments $\left\{\ell_{x}\right\}_{x \in \partial B_{1}}$ with endpoints $D v(x)$ and $D v(x)+b x$. We move these line segments to new positions, $\ell_{x} \rightarrow \tilde{\ell}_{x}$, such that one endpoint is $z_{x} \in \partial B_{R}$ and the other one is $z_{x}+b x$, and for any $x$, both $\ell_{x}$ and $\tilde{\ell}_{x}$ are on the same straight line. Then by (8.17), the area of the region covered by $\left\{\tilde{\ell}_{x}\right\}$ is larger than that of the region covered by $\left\{\ell_{x}\right\}$. Hence (8.16) holds with $c_{1}=4 \pi c_{0} R$.

By Lemma 8.2, $\Omega_{h} \subset\left\{-h \leq x_{1} \leq h\right\}$. Hence we may suppose (8.14) holds at some point $x_{0} \in\left\{x_{1}=0\right\}$, namely, $x_{0}=\left(0, a_{0}\right)$. Noting that by (8.6) and our definition of $\alpha, \Omega_{h}^{+} \subset\left\{x_{2}<C h^{1 /(1+\alpha)}\right\}$, we have

$$
a_{0} \leq C h^{1 /(1+\alpha)} \text {. }
$$


Denote $\eta(t)=v(0, t)$. Suppose $\eta^{\prime}\left(a_{0}\right) \geq 0$ (the case $\eta^{\prime}\left(a_{0}\right) \leq 0$ can be dealt with similarly). Then

$$
\eta(t)>g(t)+\frac{c_{0}}{2} h^{\alpha /(1+\alpha)}
$$

if $t \geq a_{0}$ and $g(t)<\frac{1}{2} c_{0} h^{\alpha /(1+\alpha)}$. Let

$$
h^{*}=\frac{1}{2} c_{0} h^{\alpha /(1+\alpha)} .
$$

Then (8.19) holds with

$$
a_{0} \leq t \leq\left(h^{*}\right)^{1 /\left(1+\alpha^{*}\right)},
$$

where $\alpha^{*}=\alpha\left(h^{*}\right)$.

It follows that

$$
v>u^{*} \quad \text { in } \quad G_{h^{*} / 2}:=\Omega_{h^{*} / 2} \cap\left\{x_{2}>a_{0}\right\} .
$$

Denote

$$
E=\left\{x \in \mathbf{R}^{2} \mid 2\left(x-x_{0}\right)+x_{0} \in G_{h^{*} / 2}\right\},
$$

namely, $E$ is the $\frac{1}{2}$-dilation of $G_{h^{*} / 2}$ with respect to $x_{0}$. Then by (8.6),

$$
|E| \geq C\left(h^{*}\right)^{1+1 /\left(1+\alpha^{*}\right)} \geq C_{1}|\widetilde{E}| \text {. }
$$

We also have

$$
\left|N_{u^{*}}(E)\right| \leq C\left(h^{*}\right)^{\alpha^{*} /\left(1+\alpha^{*}\right)} .
$$

To verify (8.23), one observes that $N_{u^{*}}(E)$ is contained in the set $\left\{x \in \mathbf{R}^{2}|| x_{1} \mid<\right.$ $\left.1,\left|x_{2}\right| \leq C\left(h^{*}\right)^{\alpha^{*} /\left(1+\alpha^{*}\right)}\right\}$.

Let $\hat{v}=\max \left(v, u^{*}\right)$. We claim

$$
A^{*}(\hat{v})>A^{*}\left(u^{*}\right),
$$

which implies that $u^{*}$ is not a maximizer, and so we reach a contradiction. Observing that by (8.21), $\hat{v}=v$ in $E$, and by (8.12), $\mu[\hat{v}] \geq \mu\left[u^{*}\right]$ in $B_{1}(0)-\left(E \cup \Omega_{h}\right)$, we need only to prove

$$
\int_{E}(\mu[v])^{3 / 4}>\int_{E}\left(\mu\left[u^{*}\right]\right)^{3 / 4}+\int_{\Omega_{h}^{+}}\left(\mu\left[u^{*}\right]\right)^{3 / 4},
$$

where $\mu[v]=\operatorname{det} \partial^{2} v$ and $\mu\left[u^{*}\right]=\operatorname{det} \partial^{2} u^{*}$; see (7.3). By Lemmas 2.1 and $2.3, \mu\left[u^{*}\right]$ is an integrable function.

Let $E=E_{1} \cup E_{2} \cup \Omega_{h}^{+}$, where $E_{1}=\left\{x \in E-\Omega_{h}^{+} \mid \mu\left[u^{*}\right](x)<\frac{1}{2} \delta\right\}$ and $E_{2}=E-\left(E_{1} \cup \Omega_{h}^{+}\right)$. If $\left|E_{1}\right| \geq \frac{1}{2}|E|$, then for any $x \in E_{1}$, we have

$$
(\mu[v])^{3 / 4} \geq\left(\mu\left[u^{*}\right]\right)^{3 / 4}+C \delta^{3 / 4} .
$$

On the other hand,

$$
\int_{\Omega_{h}^{+}}\left(\mu\left[u^{*}\right]\right)^{3 / 4} \leq\left|\Omega_{h}^{+}\right|^{1 / 4}\left(\int_{\Omega_{h}^{+}} \mu\left[u^{*}\right]\right)^{3 / 4}=q_{h}^{3 / 4}\left|\Omega_{h}^{+}\right|^{1 / 4} .
$$

Hence we need only

$$
\delta^{3 / 4}\left|E_{1}\right| \geq C q_{h}^{3 / 4}\left|\Omega_{h}^{+}\right|^{1 / 4}
$$

namely, $|E| \geq C\left|\Omega_{h}^{+}\right|$by (8.13) and (8.22), which is obviously true. 
Otherwise observing that $\left|\Omega_{h}^{+}\right|=o(|E|)$ as $h \rightarrow 0$, we may suppose simply that $E_{1}$ is an empty set by replacing $E$ by $E_{2}$. Then at any point $x \in E$,

$$
\begin{aligned}
\mu[v]^{3 / 4} & =\left(\mu\left[u^{*}\right]+\delta\right)^{3 / 4} \\
& \geq\left(\mu\left[u^{*}\right]\right)^{3 / 4}+C\left(\mu\left[u^{*}\right]\right)^{-1 / 4} \delta .
\end{aligned}
$$

By the Hölder inequality we have

$$
\int_{E}\left(\mu\left[u^{*}\right]\right)^{-1 / 4} \geq|E|^{5 / 4}\left[\int_{E} \mu\left[u^{*}\right]\right]^{-1 / 4} .
$$

Hence

$$
\begin{aligned}
\int_{E}(\mu[v])^{3 / 4} & \geq \int_{E}\left(\mu\left[u^{*}\right]\right)^{3 / 4}+C \delta|E|^{5 / 4}\left[\int_{E} \mu\left[u^{*}\right]\right]^{-1 / 4} \\
& =\int_{E}\left(\mu\left[u^{*}\right]\right)^{3 / 4}+C q_{h}|E|^{1 / 4}\left[\int_{E} \mu\left[u^{*}\right]\right]^{-1 / 4} .
\end{aligned}
$$

Therefore by (8.26) we need only

$$
q_{h}^{1 / 4}|E|^{1 / 4}>C\left|\Omega_{h}^{+}\right|^{1 / 4}\left[\int_{E} \mu\left[u^{*}\right]\right]^{1 / 4} .
$$

By (8.10), (8.11), (8.22) and (8.23), we need only

$$
h^{\frac{\alpha}{1+\alpha}}\left(h^{*}\right)^{1+\frac{1}{1+\alpha^{*}}} \geq C h^{1+\frac{1}{1+\alpha}}\left(h^{*}\right)^{\frac{\alpha^{*}}{1+\alpha^{*}}} .
$$

By (8.20), the above inequality holds if $\alpha<1+\alpha^{*}$, which is true by our choice of $h$ in (8.9). This completes the proof of Theorem 8.2.

Next we extend Theorem 8.2 to locally convex surfaces.

Theorem 8.3. Suppose $n=2$. Then the maximizer in Theorem 3.1 is strictly convex.

Proof. Let $\mathcal{M}$ denote the maximizer. If $\mathcal{M}$ contains a line segment $\ell$, let $P$ be a tangent plane of $\mathcal{M}$ in $\mathbf{R}^{3}$ which contains $\ell$, and let $F$ be the component of $P \cap \mathcal{M}$ which contains $\ell$. Then $F$ is a convex set 22 . If $F$ has an extreme point which is an interior point of $\mathcal{M}$, then Case (a) in the proof of Theorem 8.2 applies and we reach a contradiction.

If all extreme points of $F$ are boundary points of $\mathcal{M}$, we are in Case (b) of the proof of Theorem 8.2. In this case, by a rotation of axes we may suppose $F \subset\left\{x_{3}=0\right\}$. Then the south pole of the sphere $S^{2}$ is an interior point of the Gauss mapping image of $\mathcal{M}_{0}$. Let $F_{\delta}$ denote the connected component of $\mathcal{M} \cap\left\{x_{3}<\delta\right\}$ containing $F$. Then $F_{\delta}$ is convex if $\delta>0$ is sufficiently small [22].

Let $F_{\delta}^{-}$denote the set of points $p \in F_{\delta}$ such that the Gauss mapping $G(p)$ of $\mathcal{M}$ falls in the south hemi-sphere. Then $F_{\delta}^{-}$is the graph of an affine maximal convex function $u$. Since all extreme points of $F$ are boundary points of $\mathcal{M}$, there is a line segment $\ell \subset F$, as given in (8.1), of which both endpoints $\pm e_{1}$ are boundary points of $\mathcal{M}$. We claim that $\ell$ is transversal to $\partial \mathcal{M}$ at the endpoints $\pm e_{1}$. Indeed, extend $\mathcal{M}_{0}$ to $\widetilde{\mathcal{M}}_{0}$ such that $\widetilde{\mathcal{M}}_{0}$ is locally uniformly convex and $\mathcal{M}_{0} \cap \partial \widetilde{\mathcal{M}}_{0}=\emptyset$. Denote 
$\widetilde{\mathcal{M}}=\mathcal{M} \cup\left(\widetilde{M}_{0}-\mathcal{M}_{0}\right)$. Then $\widetilde{\mathcal{M}}$ is a locally convex hypersurface. For $\delta>0$ we denote by $\widetilde{F}_{\delta}$ the component of $\widetilde{\mathcal{M}} \cap\left\{x_{3}<\delta\right\}$ containing $\ell$. Then $\widetilde{F}_{\delta}$ contains no boundary points of $\widetilde{\mathcal{M}}$ when $\delta>0$ is small. Hence by Lemma $3.1, \widetilde{F}_{\delta}$ is convex and so $\ell$ is transversal to $\partial \mathcal{M}$.

It follows that $u$ satisfies (8.2). Let $u^{*}$ be the Legendre transform of $u$. Then $u^{*}$ satisfies (8.3) and (8.4). Hence the proof of Theorem 8.2 applies and we also reach a contradiction.

Theorem 8.3 completes the proof of Theorem A. Combining Theorem 8.2 with Theorem 6.2 and Theorems 4.1 and 4.2, we obtain for the graph case

Theorem 8.4. Let $\Omega$ be a bounded smooth domain in $\mathbf{R}^{2}$, and let $\phi \in C^{2}(\bar{\Omega})$ be a uniformly convex function. Then there exists a unique, locally uniformly convex maximizer $u \in C^{\infty}(\Omega) \cap C^{0,1}(\bar{\Omega})$ for the variational problem (1.7), (1.8).

We remark that Theorems 8.2 and 8.4 also extend to the inhomogeneous problem (1.17) for $f \in L^{\infty}(\Omega)$ with solution $u \in W_{l o c}^{4, p}(\Omega)$ for all $p<\infty\left(u \in C^{k+4, \alpha}(\Omega) \cap\right.$ $C^{0,1}(\bar{\Omega})$ if $\left.f \in C^{k, \alpha}(\Omega) \cap C^{\alpha}(\bar{\Omega})\right)$. The details will be presented in a future paper.

\section{Final REMARKS}

Remark 9.1. Let $u$ be a smooth maximizer in Theorem B. Obviously $u$ satisfies the first boundary condition $(1.9)$ and $D u(\Omega) \subset D \phi(\bar{\Omega})$. An interesting problem is whether $u$ satisfies the second boundary condition $D u(\Omega)=D \phi(\Omega)$. Recall that the Dirichlet problem of the minimal surface equation is solvable for any smooth boundary values if and only if the boundary is mean convex. Analogously for the boundary condition (1.9), additional conditions may be necessary in order that $D u(\Omega)=D \phi(\Omega)$ holds.

In 19 we studied the regularity of hypersurfaces $\mathcal{M}_{\Gamma}$ contained in a given closed, uniformly convex hypersurface $\Gamma$ with maximal affine area, and we proved that the contact set $\mathcal{M}_{\Gamma} \cap \Gamma$ must be affine mean convex. Therefore a reasonable assumption such that $D u(\Omega)=D \phi(\Omega)$ holds is that $\phi$ is affine mean convex, namely, the affine mean curvature of the graph of $\phi, H_{A}[\phi]=-\frac{1}{n+1} L[\phi]$, is positive.

Remark 9.2. In [21] we proved that the function $u$ in $\mathbf{R}^{10}$, given by

$$
u(x)=\sqrt{\left|x^{\prime}\right|^{9}+x_{10}^{2}},
$$

where $x^{\prime}=\left(x_{1}, \cdots, x_{9}\right)$, is affine maximal. Therefore in high dimensions $(n \geq 10)$ there exist affine maximal functions vanishing on the boundary of a convex domain which are not strictly convex. We have not found similar examples in dimensions $3 \leq n \leq 9$.

Remark 9.3. Let $u$ be a convex solution to the Monge-Ampère equation

$$
\operatorname{det} D^{2} u=1 \text {. }
$$

Then $u$ satisfies the affine maximal surface equation (1.5). When $n \geq 3$, there exist nonsmooth convex solutions to the above Monge-Ampère equation [17]. Hence there exist nonsmooth affine maximal hypersurfaces when $n \geq 3$. 
However a convex solution to the general Monge-Ampère equation

$$
\operatorname{det} D^{2} u=f \quad \text { in } \Omega \text {, }
$$

where $f$ is a bounded, positive function, is strictly convex in any dimension if $u$ vanishes on $\partial \Omega$ or both $\partial \Omega$ and the trace of $u$ on $\partial \Omega$ are smooth. For the affine maximal surface equation, an interesting question is whether the maximizer $u$ in Theorem B is strictly convex if $\phi$ is uniformly convex.

\section{REFERENCES}

[1] W. Blaschke, Vorlesungen über Differential geometrie, Berlin, 1923.

[2] L.A. Caffarelli, A localization property of viscosity solutions to the Monge-Ampère equation and their strict convexity, Ann. Math., 131(1990), 129-134. MR1038359 (91f:35058)

[3] L.A. Caffarelli, Interior $W^{2, p}$ estimates for solutions of Monge-Ampère equations, Ann. Math., 131(1990), 135-150. MR1038360 (91f:35059)

[4] L.A. Caffarelli and C.E. Gutiérrez, Properties of the solutions of the linearized MongeAmpère equations, Amer. J. Math., 119(1997), 423-465. MR1439555 (98e:35060)

[5] L.A. Caffarelli, L. Nirenberg, and J. Spruck, The Dirichlet problem for nonlinear second order elliptic equations I. Monge-Ampère equation, Comm. Pure Appl. Math., 37(1984), 369-402. MR0739925 (87f:35096)

[6] E. Calabi, Hypersurfaces with maximal affinely invariant area, Amer. J. Math., 104(1982), 91-126. MF 0648482 (85b:53054)

[7] E. Calabi, Affine differential geometry and holomorphic curves, Lecture Notes Math., 1422(1990), 15-21. MR1055839 (91c:53009)

[8] S.Y. Cheng and S.T. Yau, Complete affine hypersurfaces, I. The completeness of affine metrics, Comm. Pure Appl. Math., 39(1986), 839-866. MR0859275 (87k:53127)

[9] S.S. Chern, Affine minimal hypersurfaces, in Minimal submanifolds and geodesics, Proc. Japan-United States Sem., Tokyo, 1977, 17-30. MR0574250 (81e:53007)

[10] D. Gilbarg and N.S. Trudinger, Elliptic partial differential equations of second order, Springer-Verlag, New York, 1983. MR0737190 (86c:35035)

[11] B. Guan and J. Spruck, Boundary value problems on $S^{n}$ for surfaces of constant Gauss curvature, Ann. of Math., 138(1993), 601-624. MR1247995 (94i:53039)

[12] N. Ivochkina, A priori estimate of $\|u\|_{C^{2}(\bar{\Omega})}$ of convex solutions of the Dirichlet problem for the Monge-Ampère equation, Zap. Nauchn. Sem. Leningrad. Otdel. Mat. Inst. Steklov. (LOMI), 96(1980), 69-79 (Russian). English translation in J. Soviet Math., 21(1983), 689697. MR0579472 (82b:35056)

[13] N.V. Krylov, Nonlinear elliptic and parabolic equations of the second order, Reidel, Dordrecht-Boston, 1987. MR.0901759 (88d:35005)

[14] K. Leichtweiss, Affine geometry of convex bodies, Johann Ambrosius Barth Verlag, Heidelberg, 1998. MR1630116 (2000j:52005)

[15] E. Lutwak, Extended affine surface area, Adv. Math., 85(1991), 39-68. MR1087796 (92d:52012)

[16] K. Nomizu and T. Sasaki, Affine differential geometry, Cambridge University Press, 1994. MF,1311248(96e:53014)

[17] A.V. Pogorelov, The muitidimensional Minkowski problems, J. Wiley, New York, 1978. MR 0478079 (57:17572)

[18] M.V. Safonov, Classical solution of second-order nonlinear elliptic equations, Izv. Akad. Nauk SSSR Ser. Mat., 52(1988), 1272-1287 (Russian). English translation in Math. USSRIzv., 33(1989), 597-612. MR0984219 (90d:35104)

[19] W.M. Sheng, N.S. Trudinger, and X.-J. Wang, Enclosed convex hypersurfaces with maximal affine area, preprint.

[20] U. Simon, Affine differential geometry, in Handbook of differential geometry, North-Holland, Amsterdam, 2000, 905-961. MR1736860 (2001c:53014)

[21] N.S. Trudinger and X.-J. Wang, The Bernstein problem for affine maximal hypersurfaces, Invent. Math., 140(2000), 399-422. MR.1757001 (2001h:53016)

[22] N.S. Trudinger and X.-J. Wang, On locally convex hypersurfaces with boundary, J. Reine Angew. Math., 551(2002), 11-32. MR1932171(2004b:58017) 
[23] N.S. Trudinger and X.-J. Wang, On boundary regularity for the Monge-Ampère and affine maximal surface equations, preprint.

[24] X.-J. Wang, Affine maximal hypersurfaces, Proc. ICM, Vol. III, 2002, 221-231. MR 1957534 (2004j:35110)

[25] W. P. Ziemer, Weakly differentiable functions, Springer, 1989. MR.1014685 (91e:46046)

Centre for Mathematics and Its Applications, The Australian National University, Canberra, ACT 0200, Australia

E-mail address: Neil.Trudinger@maths.anu.edu.au

Centre for Mathematics and Its Applications, Australian National University, CanBerra, ACT 0200, Australia

E-mail address: X.J.Wang@maths.anu.edu.au 